\title{
Intelligent Techniques Using Molecular Data Analysis in Leukaemia: An Opportunity for Personalized Medicine Support System
}

\author{
Haneen Banjar, ${ }^{1,2}$ David Adelson, ${ }^{3}$ Fred Brown, ${ }^{1}$ and Naeem Chaudhri ${ }^{4}$ \\ ${ }^{1}$ School of Computer Science, University of Adelaide, Adelaide, SA, Australia \\ ${ }^{2}$ Department of Computer Science, King Abdulaziz University, Jeddah, Saudi Arabia \\ ${ }^{3}$ School of Molecular and Biomedical Science, University of Adelaide, Adelaide, SA, Australia \\ ${ }^{4}$ Oncology Centre, Section of Hematology, HSCT, King Faisal Specialist Hospital and Research Centre, Riyadh, Saudi Arabia
}

Correspondence should be addressed to Haneen Banjar; hrbanjar@kau.edu.sa

Received 5 April 2017; Revised 12 June 2017; Accepted 15 June 2017; Published 25 July 2017

Academic Editor: Junya Kuroda

Copyright (C) 2017 Haneen Banjar et al. This is an open access article distributed under the Creative Commons Attribution License, which permits unrestricted use, distribution, and reproduction in any medium, provided the original work is properly cited.

\begin{abstract}
The use of intelligent techniques in medicine has brought a ray of hope in terms of treating leukaemia patients. Personalized treatment uses patient's genetic profile to select a mode of treatment. This process makes use of molecular technology and machine learning, to determine the most suitable approach to treating a leukaemia patient. Until now, no reviews have been published from a computational perspective concerning the development of personalized medicine intelligent techniques for leukaemia patients using molecular data analysis. This review studies the published empirical research on personalized medicine in leukaemia and synthesizes findings across studies related to intelligence techniques in leukaemia, with specific attention to particular categories of these studies to help identify opportunities for further research into personalized medicine support systems in chronic myeloid leukaemia. A systematic search was carried out to identify studies using intelligence techniques in leukaemia and to categorize these studies based on leukaemia type and also the task, data source, and purpose of the studies. Most studies used molecular data analysis for personalized medicine, but future advancement for leukaemia patients requires molecular models that use advanced machine-learning methods to automate decision-making in treatment management to deliver supportive medical information to the patient in clinical practice.
\end{abstract}

\section{Introduction}

Molecular cytogenetics of hematological malignancies and therapies is under development. Leukaemia is a hematological disorder where two leukaemia patients who may appear identical morphologically may have different molecular profiles and thus the variation in response to the prescribed therapies would be unpredictable [1]. Leukaemia usually begins in the bone marrow, which is the site where all blood cells are formed and produced. When a person has leukaemia, the white blood cells produced are usually numerous, and they are abnormal, which means that they cannot effectively defend the body from diseases, pathogens, or foreign substances. The type of white blood cells affected, either lymphoid or myeloid, can identify the type of leukaemia.
Four common types of leukaemia are chronic lymphocytic leukaemia (CLL), chronic myeloid leukaemia (CML), acute lymphocytic leukaemia (ALL), and acute myeloid leukaemia (AML) [2].

The most common modes of treatment for leukaemia involve chemotherapy, radiation therapy, stem cell transplantation, and immunotherapy with interferon [2]. Many patients become disease-free after years of treatment, proceeding to live normal lives. However, these modes of therapy can have disastrous consequences for the victims of leukaemia. For instance, chemotherapy often makes patients lose their hair, it can darken their skin, and it can cause infertility in young patients. Bone marrow transplantation is also very expensive, and it is not often easy to find a matching bone marrow donor, especially if close family members 
are not a match. The pharmacogenomics (PGx) aims to replace general modes of treatment, such as chemotherapy and bone marrow transplantation, adopting instead a tailormade course of medication designed according to a patient's particular genetic makeup [3]. Although multiple targeted therapies are available to use for leukaemia patients [4], it is difficult to select among the available targeted therapies.

Therefore, the use of intelligent techniques in medicine has brought a ray of hope in terms of treating leukaemia patients. Intelligent techniques are able to conduct automatic searches to discover knowledge and learn from data to facilitate the task and achieve the objective. The broad areas frequently defined under intelligence techniques are as follows: knowledge discovery, machine learning, and data mining. These areas use statistics and probability to detect patterns that are difficult to study manually. Intelligent techniques will integrate various molecular technologies and sources of data, information, or knowledge to facilitate the development of personalized medicine and decision-making by physicians.

The personalized decision support system requires personal information or genetic information, such as genetic tests and medical tests, for each patient to integrate, as far as possible, the knowledge gained from genomics research relating to the disease in question [5]. From this definition, it is clear that the need for personalized decision support systems in leukaemia treatment has increased because of the massive amount of available genetic and genomic information. With the use of the personalized medicine support system, leukaemia treatment will no longer be a trial and error game and it will be possible to select which drugs will work at which stage. The outcomes are expected to provide a preventive, next-generation therapy, with better specific interventions for individual patients.

Personalized medicine support systems can use available knowledge resources to deliver just-in-time information to individualize therapy. The existing pharmacogenomics knowledge base (PharmGKB) (available at http:// www.pharmgkb.org) is a massive resource that provides researchers with information relevant to genetic variations on drug responses. The second source is to translate PGx knowledge into a rule-based representation where the rules are extracted from the characteristics of PGx knowledge in the US Food and Drug Administration (FDA) drug label database [6]. Another knowledge resource is the Clinical Practice Guidelines (CPG) document, which lists a set of guidelines for cases with specific diagnoses, along with recommended therapeutic action plans [7].

Developing personalized medicine support systems in some medical applications has already made significant progress. First, in cardiovascular diseases, many factors could influence cardiovascular disease, such as genes, environment, and lifestyle (exercise and nutrition). It was important to develop models for prevention, treatment management, or detecting disease to assist clinicians in treating cardiovascular patients. Indeed, the personalized decision support system for cardiovascular patients was constructed using two models. One model was for risk assessment using patients' personal information, and the other was for generating advice to clinicians based on the first model's results [8]. The second application was in type 1 diabetes mellitus. The frequent measurements of glucose levels, monitoring physical activity, and personal information about the genome, such as the genes that could cause obesity and predisposition for diabetes, were used to create a personalized decision support system for treating, preventing, and monitoring patients [9]. Another system for optimizing insulin infusion rates based on nonlinear model predictive control was designed using in silico data from a virtual type 1 diabetes patient, where the system was evaluated using data from a mathematical model of a patient with type 1 diabetes. The results showed the effectiveness of using such methods with diabetes patients [10]. The third application is colon cancer, where selection of the treatment plan was the objective of the personalized medicine support system. The clinical and genomic features were used for early diagnosis in colon cancer using cluster techniques. The platform known as MATCH supported clinicians in making decisions about patients with colon cancer [11]. Adding genetic features improves diagnosis compared to previous research methods. Further methods proposed for colon cancer prediction based on genetic information were studied by Kulkarni et al. [12]. The last example, but not limited to those mentioned in our review, breast cancer classification is an active research application in which the personalized medicine support medicine was the objective in multiple studies [13-31].

One angle of personalized medicine is to identify the correct disease subtype and patient classification. Machinelearning techniques were proven to achieve a high performance classification in identifying patient subtypes by using a support vector machine (SVM) and uncertainty SVM [32], in predicting drug sensitivity in cancer by using Bayesian networks $[33,34]$, in predicting patient response to therapy by using ensemble methods [13], in predicting risk category using soft computing methods [35], or in recommendations to enhance lifestyle and educate patients about healthy solutions [36]. Thus, intelligent methods should be proposed to create a personalized medicine support system in leukaemia. These methods required information about preknowledge in allocating optimal treatment, responding to each patient's risk category, which should be provided to evaluate the models.

Medical researchers continue to emphasise that their studies are updated with the most effective treatment protocols, which could be used to treat leukaemia patients. The current system for achieving personalized medicine in leukaemia has been established by using the predictive factors to determine upfront treatment. Many groups of researchers have conducted studies by using different techniques to investigate several factors that could affect the drug responses. Studying a single biomarker as a predictive substance could indicate the response pretreatment and predict the risk to the individual [37]. The other approach involves predicting the drug reaction in terms of toxicity or resistance, using an individual's genotype data and clinical data to improve the individual's care [38]. A pharmacogenetics is a field that studies the individual's response to a specific therapy based on the person's genotype information. With the human 
molecular tests and the development of molecularly targeted therapy, the system for achieving personalized medicine for leukaemia has additional levels of information that needs to be processed with assistance from information technology.

According to current knowledge, many leukaemia researchers have applied intelligent techniques, but no reviewers have yet undertaken a systematized literature review from a computational perspective concerning the development of personalized medicine intelligent techniques for leukaemia patients using molecular data analysis. This review studies the published empirical research on personalized medicine in leukaemia and synthesizes findings across studies related to intelligence techniques in leukaemia, with specific attention to particular categories of these studies to help identify opportunities for further research into personalized medicine support systems in one category of leukaemia, namely, chronic myeloid leukaemia. A systematic search was carried out to identify studies using intelligence techniques in leukaemia and to categorize these studies based on leukaemia type and also the task, data source, and purpose of the studies. Our review is conducted to support health informatics and biomedical and bioinformatics in order to answer specific technical questions to help develop future research into leukaemia from a technical perspective.

\section{Methods}

2.1. Search Strategy. Ten databases were searched, including Scopus, PubMed, Web of Science, BIOSIS, Inspec, MEDLINE, Embase, Springer, ACM Digital Library, and IEEE Xplore. The review was restricted to English-language studies published from 2001 to October 2016 because, prior to 2001, molecular targeted therapies and molecular responses for personalized medicine were not approved by the FDA for medical treatments in leukaemia but became more popular around this time [39]. The search was limited to primary research articles. The eligibility of each study was evaluated based on the title and abstract. Only full-text articles were retrieved. The terms searched were leukaemia and leuk ${ }^{*}$, with different combinations of key words for intelligent systems and techniques (machine learning, data mining, knowledge extraction, and CDS system) and with combined keywords and/or subject headings to identify technical leukaemia articles. Since studies were screened by a single researcher and then reviewed by the coauthors, this work cannot be considered a systematic review, but it could be considered a "systematized review" [40] to demonstrate comprehensive search guidelines and an elaborative quality assessment and synthesis of research evidence.

2.2. Selection of Studies and Data Extraction. The resulting abstracts were evaluated for inclusion. Then, the full text of those identified as meeting the criteria were obtained. Studies were included in the review, if the study

(i) used molecular data from adult leukaemia patients;

(ii) used intelligence techniques to achieve the purpose of the study; (iii) was implemented as a model for adult leukaemia patients;

(iv) was published in a peer-reviewed journal between 2001 and 2016;

(v) was published in English.

Because the intention was to review the literature to identify whether opportunities currently under clinical development are related to model analysis molecular data for personalized medicine in leukaemia, articles were excluded, if they

(1) published decision-analytic models for economic purposes;

(2) used pediatric leukaemia data;

(3) studied a nonpatient population;

(4) were not written in English;

(5) were doctoral dissertations or pilot studies;

(6) did not include the full text of the study report.

\section{Results}

3.1. Study Selection. In total, $n=1,929$ citations were retrieved. Excluding duplicates, the search yielded $n=1,747$ articles, and the initial screen of abstracts yielded $n=745$ articles to undergo a full-text review. Ultimately, 55 studies met the eligibility criteria and underwent data extraction and analysis (Figure 1).

55 studies described 55 unique intelligent techniques (Table 2). The studies were analyzed based on the leukaemia type involved in the study, the task, the data source, and the purpose of the study.

3.2. Based on Leukaemia Type. Of the commonest leukaemia types (Figure 2) involved in previous studies, AML and ALL occupied most of the classification studies because the DNA microarray data can be downloaded online from the Cancer Program Legacy Publication Resources [41].

Some studies [42-44] distinguished ALL origin cell lines from non-ALL leukaemia origin cell lines. A study [45] demonstrated a decision support system that classifies all four types of leukaemia using principal components for feature selection and clustering. A few studies involved chronic leukaemia types to identify the molecular biomarker in CML. For example, Oehler et al. [46] used Bayesian model averaging, while Yeung et al. [47] integrated expert knowledge to predict the functional relationships in gene expression. The capture of disease pathophysiology across patient types was studied by Savvopoulos et al. [48], and temporally and spatially distributed models were built to extract knowledge from CLL patients' blood samples. In CML and CLL studies, some studies were not included in the final selection because most of these studies were prospective studies ending with a description of patient population outcomes, rather than building models or using intelligent techniques. A study of combined prognostic markers using a multivariate model for knowledge extraction is included in this study because it 


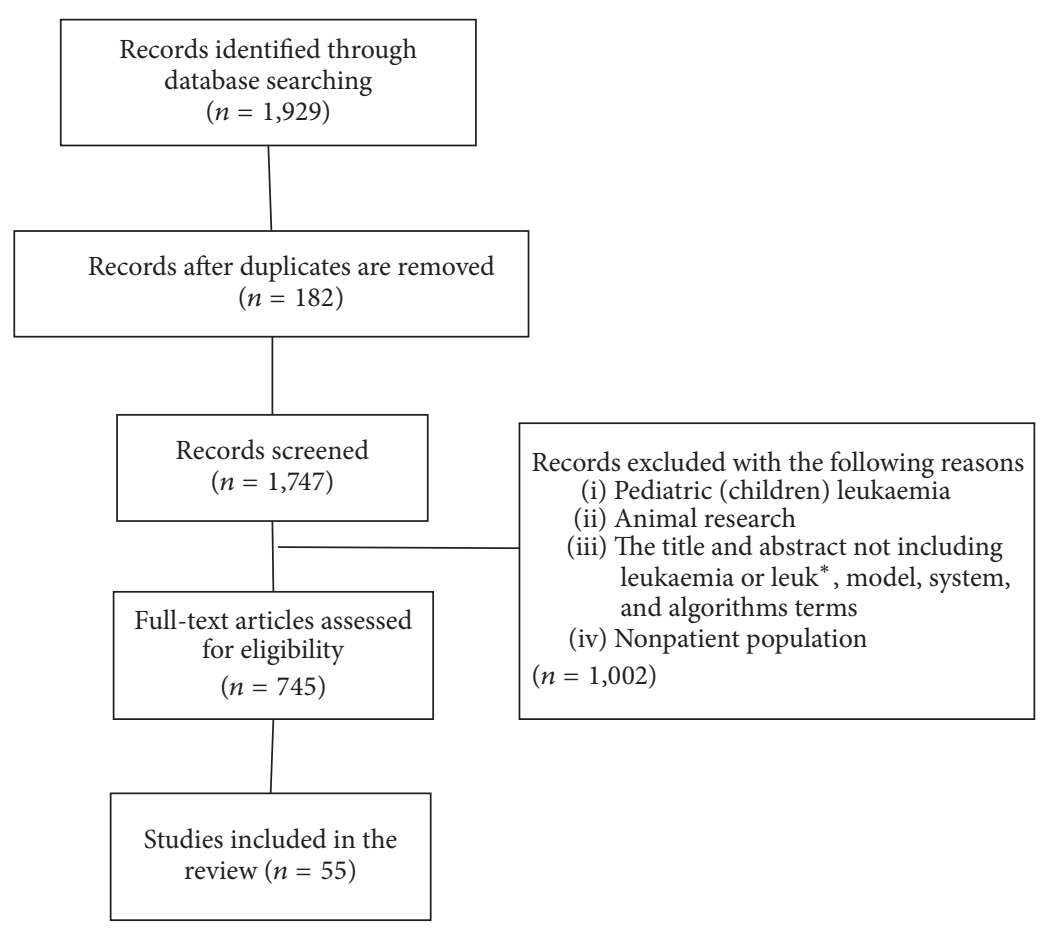

FIGURE 1: Flow chart showing the article-selection process.

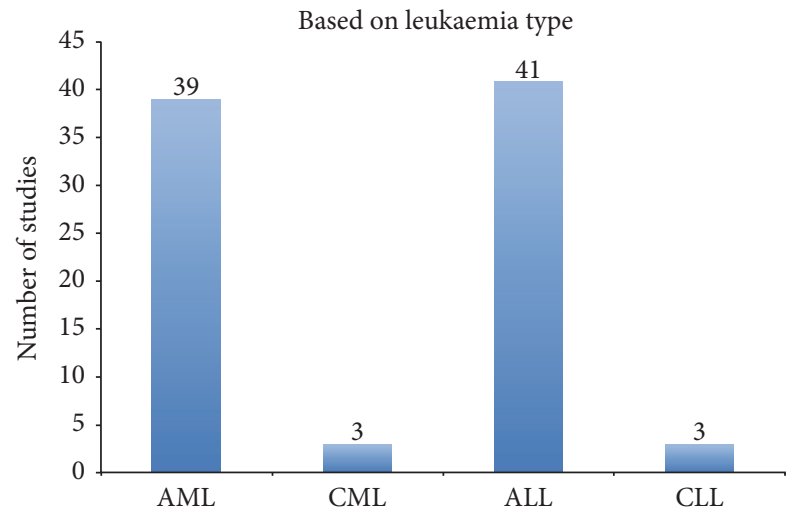

FIGURE 2: Summary of the frequency of studies based on leukaemia type.

proved that integration of gene expression in a model can predict outcomes in CLL [49].

Emphasis has been placed on CML as a research opportunity because of developments in monitoring CML patients' molecular response to molecular targeted therapy. The Australian Institute of Health and Welfare (AIHW) classified myeloid cancers as the 9th most commonly diagnosed cancer in 2016, with around 3,624 cases in Australia [50]. Chronic myeloid leukaemia is also known as chronic myelogenous leukaemia or chronic granulocytic leukaemia. White blood cells are affected when the bone marrow produces an unusual number of white blood cells. These cells enter the bloodstream and accumulate in organs such as the spleen or liver. If the disease progresses, the bone marrow could produce an excessive number of immature white blood cells. Consequently, the bone marrow cannot make enough red cells, normal white cells, and platelets [51].

In CML, according to White and Hughes [52], the previous studies did not establish criteria for selecting the best molecular targeted therapy for each patient, particularly following the availability of multiple therapies that can represent a perfect application of personalized therapy based on predicting patients' molecular response to molecular target therapy.

3.3. Based on Data Source. Microarray technology is an area of considerable focus for the purpose of cancer diagnosis (Figure 3). One study [45] used exon arrays to classify patients who suffer from different forms of leukaemia at various stages. Another study used human leukaemia tissue to determine different cluster differentiation (CD) markers [53]. Although seven existing studies used bone marrow cell images to classify leukaemia subtype, microarrays are still used to facilitate different types of experiments and clarify the results, making it easy for researchers to propose molecular medicines in contrast to the rate of tumor progression. The other example used gene-expression profiling among adult patients, which proved crucial in treating leukaemia. The researchers stated that, with the help of expression profiling, doctors are able to determine how a patient adapts to treatment methods. In addition, based on these facts, a physician is able to determine the survival probability of the patients in question. 


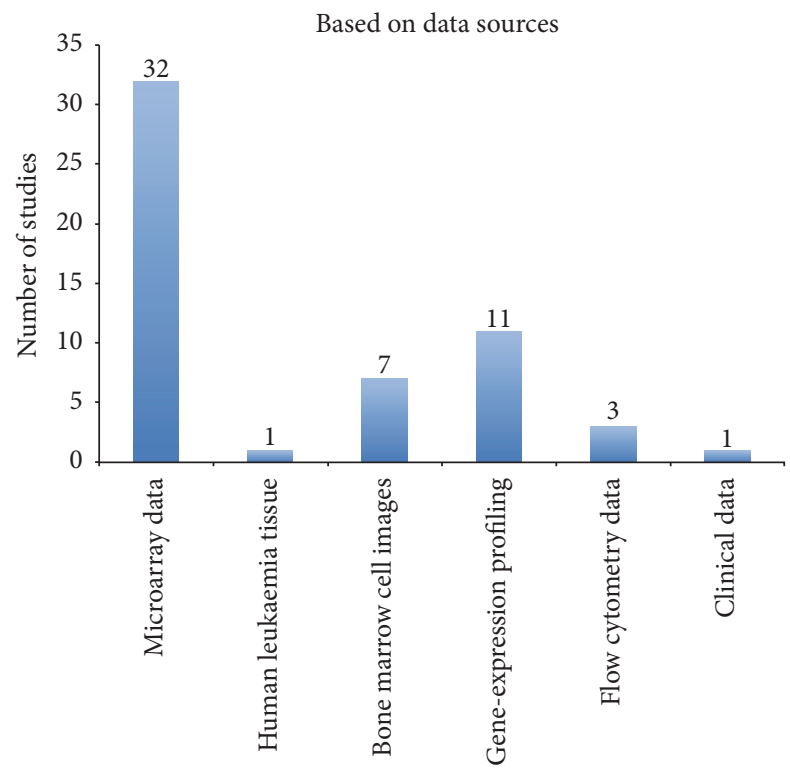

Figure 3: Summary of the frequency of studies based on data sources.

A huge opportunity arises from integrating data sources such as image data, clinical data, lifestyle or family history, SNP, gene-expression profiles, proteomics profiles, and metabolomics profiles. For example, SNPs have been investigated in an attempt to determine the susceptibility rate of patients suffering from leukaemia, which can support cases where patients have been diagnosed with leukaemia. The use of SNPs made it possible for physicians to predict the likely survivability of their patients after treatment, which is useful in determining the most suitable medical interventions.

In terms of patient care and administration, electronic health records (EHR) are often reused in research to answer specific research questions $[5,54-56]$. Cases are matched with enquiries based on obtained research criteria for patient inclusion, and a dataset of many matches can then be generated for analysis. The EHR may include sparse data or missing values, as some patients may not seek frequent care. The quality of EHR would likely impact the bias of research findings or modeling performance. Derivation of key variables is also an important aspect when dealing with EHR, as the values may be recorded in different ways in different systems. This arises due to varying definitions between sources. The quality of data and correct values of derived key variables are of concern to researchers, but many algorithms can be investigated during preprocessing procedures to improve the quality of data, which would possibly lead to more reliable results [57].

$\mathrm{Yu}$ et al. [58] also divided the source of data and knowledge into three sources: clinical trial, systems biology, and healthcare systems. The meta-analysis and systematic reviews from published clinical trials are the main sources for gathering data and knowledge, although these methods have limitations over time. The difficulty of refining knowledge as new knowledge arises is an issue, as is the length of time required to build a knowledge base using systematic review and meta-analysis. The second source is system biology. The huge amount of data and knowledge collected as panomics for oncology patients come from genomics, transcriptomics, proteomics, and metabolomics data. For example, the Global Alliance for Genomics and Health [59] provides terabytes of genomic and clinical data for researchers. The third source is healthcare systems that provide knowledge in digital formats.

The other important source that has not attracted much interest in leukaemia studies is the data resulting from clinical trials studying healthy populations or epidemiological studies. Future development of clinical decisions can be guided by lessons learned from previous trials. Late-phase clinical trials (phases II, III, and IV) are considered to be massive sources of information that can be used to build personalized models. There is also a rapid increase in the number of electronic medical research databases that provide an opportunity for researchers to reuse medial data to create mathematical models.

The NCI [60] is a US agency that lists ongoing clinical trials that are testing molecular target therapies, including most of the studies conducted by investigators at hospitals and medical centers. The NCI offers full trial descriptions and names of principal investigators, so researchers can contact investigators and collaborate to conduct the proposed research.

The issue with the clinical trial data that it may be biased in several aspects: sampling, referral, selection, method, and clinical spectrum biases. Clinical trials may use sampling methods, sample size, and inclusion and exclusion criteria. Another aspect is referral bias where patients are referred by specialists and the data will represent preselected patients who have high prevalence of disease. Selection bias is clear when the clinical trial data includes groups based on a variety of demographics. In the method aspect, the data may be collected using different measurements, which leads to varying precision and specifications. Finally, the clinical spectrum bias represented in patient records may show other medical problems apart from the disease [61]. For instance, Saussele and Pfirrmann [62] have reported clinical trials in CML. They demonstrated several aspects that may cause challenges to reusing clinical trials. According to Saussele and Pfirrmann, the definition of "remission" varies in clinical trials by major molecular response (MMR) or complete cytogenetic response (CCR). In addition, the clinical trials used different primary endpoints such as 12 months' MMR or 12 months' CCR to judge treatment success. The American Society of Hematology (ASH) has established annual meetings to discuss medical trial outcomes. In 2006, the 48th ASH Annual Meeting [63] displayed a poster about the International Randomized Study of Interferon versus Imatinib STI571 (IRIS) trial. This study showed that Imatinib is appropriate for CML patients in the chronic phase as results indicate that patients receiving long-term (5-6 years) Imatinib therapy achieve MMR in $90 \%$ of cases [64]. For CML patients, these results show the efficacy of continuing to receive Imatinib over time. In 2010, two significant medical trials, DASISION (Dasatinib versus Imatinib Study in Treatment-Naïve CML) and ENESTnd (Evaluating Nilotinib Efficacy and Safety in Clinical Trials-Newly Diagnosed Patients), were discussed 
at the 52nd Annual Meeting [65]. In DASISION [66], 259 patients were studied for their response to $100 \mathrm{mg}$ Dasatinib once daily versus 260 patients who consumed $400 \mathrm{mg}$ of Imatinib once daily. The MMR was lower for the Imatinib set compared to the Dasatinib set. The most important result was the safety profiles of these drugs, which were similar. The ENESTnd trial focused on the comparison of Nilotinib versus Imatinib [67]. The samples were newly diagnosed CML-CP patients. In the trial, 282 patients were given $300 \mathrm{mg}$ Nilotinib twice daily, 281 patients received $400 \mathrm{mg}$ Nilotinib once daily, and 283 patients received $400 \mathrm{mg}$ Imatinib once daily; the median follow-up for these patients was 18 months. The cytogenetic response (CCyR) and MMR were lower in patients treated with Imatinib than in patients treated with Nilotinib. From these trial results, the debate about selecting the optimal TKI to achieve optimal patient response has been established with recognition of the need for a predictive assay that could predict the patient's response to initial treatment. The researchers also recognize the need for a medical trial to compare each TKI against the others as a first-generation treatment. The introduction of molecular targeted drugs (TKIs) has led to a dramatic improvement in the lifespan of patients affected by this condition. With the three common TKIs, Imatinib, Nilotinib, and Dasatinib currently approved to use as frontline therapy, an important question arises regarding which TKI should be prescribed. White and Hughes [52] stated that, with the lack of clear recommendations about which TKI to select, clinicians may prescribe a particular TKI based on their own preference. It is possible to extract knowledge and modeling systems using medical data and knowledge in leukaemia, but it requires advanced computational methods, such as intelligent systems. Using the available data and knowledge to construct a personalized medicine support system for leukaemia may provide massive amounts of information to use for evaluating therapies and also for potential diagnostic and prognostic markers.

3.4. Based on the Purpose of the Studies. Medical research studies have several purposes, including classification of cancer types or distinguishing healthy cells from unhealthy cells for the purpose of diagnosis, identifying markers to help in the management of treatment, and determining the prognosis of risk. Managing leukaemia patients has gained attention since a successful study by Alvey et al. [68], who developed an expert system by using a tree-structured logical program and produced over 700 clinical diagnostic rules. Even though this study focused on a specific diagnostic aspect of the system, another study [69] provided information for managing patients from registration to diagnosis and through follow-up after treatment. The study integrated history, physical examinations, and laboratory data to develop a decision support system for leukaemia diagnostics. Chae et al. [69] used profiles from patients admitted to Severance Hospital in Seodaemun District, Republic of Korea, and used data from over 490 patients to discover knowledge that helped physicians in decision-making. There is some evidence that interdisciplinary cooperation between biologists, medical scientists, computer scientists, and engineers can be

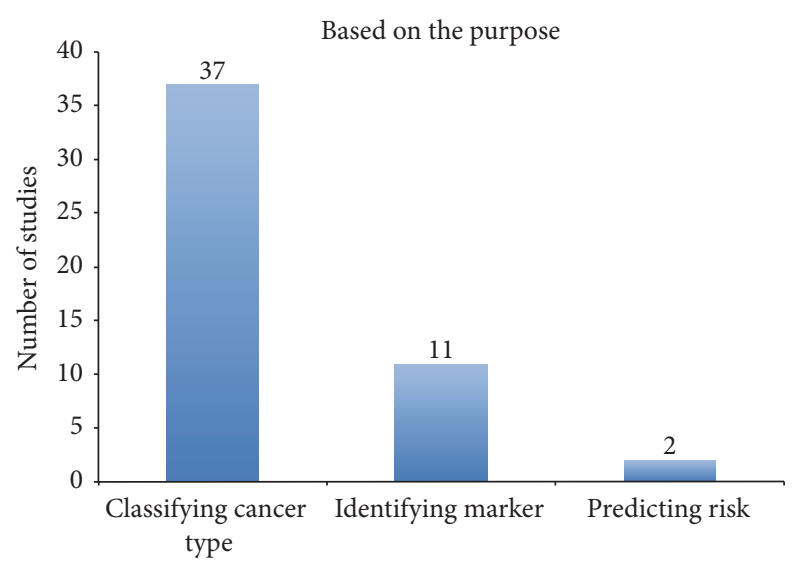

FIGURE 4: Summary of the frequency of studies based on the purpose of the studies.

productive. However, this research team did not incorporate molecular data in their system.

Among the 55 studies (Figure 4), the common purpose for conducting the studies was classifying the cancer type or subtype, with the aim of diagnosing leukaemia patients. An identifying marker in support of treatment management was observed in 11 studies. The other purpose for conducting the studies was using intelligent techniques and statistical methods for prognosis [70-73]. For example, predicting a relapse prior to transplantation in CML by integrating iterative Bayesian model averaging includes expert knowledge and expected functional connections in expression analyses in order to recognize genes causative of CML evolution [47]. This kind of model provides high-quality results, especially in complex diseases, but has varying levels of classification precision.

A new development is to extract relationships between biomarkers and the outcome in leukaemia patients. Focusing on CML, a predictive factor is a patient characteristic used to predict response to treatment [74]. The predictive factors related to the MMR response include common molecular assays. Other factors depend on peripheral blood counts as well as on the molecular-based and clinical observations of individual patients. In order to select the most effective TKI therapy at the time of diagnosis, various predictive factors in CML have been investigated to distinguish patients at an increased risk of failure with Imatinib, the first-generation TKI [52, 75-77]. Table 1 shows the current predictive assays and score systems, the factors included in the score systems and the methods used, the target prediction, and the published results.

Many studies $[77,129]$ have shown that predictive factors could probably assist in predicting patient response. Milojkovic et al. [129] conducted their study in order to predict success or failure of treatment with second-generation TKIs in CML patients using univariate analyses. They analyzed a cohort of $80 \mathrm{CML}$ patients in the first chronic phase who were treated with Dasatinib or Nilotinib. Their score system predicted the probability of CML patients achieving a CCR. The system was based on three factors: cytogenetic 
TABLE 1: The current methods used to identify risk in CML.

\begin{tabular}{|c|c|c|c|c|}
\hline \multicolumn{5}{|c|}{ Previous methods } \\
\hline Study & Factors & Method & Target prediction & Data and results \\
\hline Sokal score, Sokal et al. [78] & $\begin{array}{l}\text { Age, spleen size } \\
(\mathrm{cm}) \text {, blast }(\%) \\
\text { and platelets } \\
\left(10^{9} / \mathrm{L}\right)\end{array}$ & $\begin{array}{c}\text { Multivariate } \\
\text { analysis of survival }\end{array}$ & $\begin{array}{l}\text { Risk groups for } \\
\text { chemotherapy }\end{array}$ & $\begin{array}{c}\text { Six European and } \\
\text { American sources } \\
(n=813), \text { low } 39 \%, \\
\text { intermediate } 38 \% \text {, and high } \\
23 \%\end{array}$ \\
\hline $\begin{array}{l}\text { Hasford score, Hasford et } \\
\text { al. [79] }\end{array}$ & $\begin{array}{l}\text { Age, spleen size } \\
(\mathrm{cm}), \text { blasts }(\%), \\
\text { eosinophils }(\%), \\
\text { basophils }(\%), \text { and } \\
\text { platelets }\left(10^{9} / \mathrm{L}\right)\end{array}$ & $\begin{array}{l}\text { Multivariate } \\
\text { analysis of survival }\end{array}$ & $\begin{array}{l}\text { Risk groups for } \\
\text { interferon alpha } \\
\text { alone }\end{array}$ & $\begin{array}{c}14 \text { studies }(n=981), \text { low } \\
40.6 \% \text {, intermediate } 44.7 \% \text {, } \\
\text { and high } 14.6 \%\end{array}$ \\
\hline $\begin{array}{l}\text { EUropean Treatment } \\
\text { Outcome Study (EUTOS) } \\
\text { Score, Hasford et al. [80] }\end{array}$ & $\begin{array}{l}\text { Basophils }(\%) \text { and } \\
\text { spleen size }(\mathrm{cm})\end{array}$ & $\begin{array}{c}\text { Multivariate } \\
\text { analysis of } \\
\text { response }\end{array}$ & $\begin{array}{l}\text { CCgR at } 18 \text { months } \\
\text { to Imatinib }\end{array}$ & $\begin{array}{c}\text { Five national study groups } \\
(n=2,060), \text { low } 79 \% \text { and } \\
\text { high } 21 \%\end{array}$ \\
\hline $\begin{array}{l}\text { EUTOS Long-Term } \\
\text { survival (ELTS) score, } \\
\text { Hoffmann et al. [81] }\end{array}$ & $\begin{array}{l}\text { Age, spleen size } \\
(\mathrm{cm}), \text { blast }(\%), \\
\text { and platelets } \\
\left(10^{9} / \mathrm{L}\right)\end{array}$ & $\begin{array}{l}\text { Multivariate } \\
\text { analysis of } \\
\text { response }\end{array}$ & Long-term survival & $\begin{array}{c}(n=2,205) \text { low } 61 \% \text {, } \\
\text { intermediate } 27 \% \text {, and high } \\
12 \%\end{array}$ \\
\hline
\end{tabular}

response to Imatinib, Sokal score, and recurrent neutropenia during Imatinib treatment. Although this study used simple statistical methods, the system succeeded in classifying three risk categories: good, intermediate, and poor risk. In addition, Jabbour et al. [77] also studied the factors for predicting 123 CML patients' response after Imatinib failure. The variables used in this study included sex, CML duration, months' performance status, splenomegaly, prior interferon therapy, peripheral blood, bone marrow, best cytogenetic response to Imatinib, second-generation Nilotinib or Dasatinib therapy, active disease at the start of the second course of TKIs, clonal evaluation, higher than $90 \% \mathrm{Ph}$ positivity, and IC50 for Nilotinib and Dasatinib for in vitro inhibition of kinase activity of the mutated point in $B C R-A B L$. They also used univariate and multivariate analyses, such as the logistic regression model and the Cox proportional hazard model, in order to identify prognostic factors associated with MCyR and survival, and they succeeded in identifying three risk groups: low, intermediate, and high risk.

Previously, two of the predictive factors closely involved in predicting the molecular response in CML were identified. The first such factor is IC50. In 2005, White et al. [130] studied inhibitory concentration $50 \%$ (IC50 $0^{\text {imatinib }}$ ) as a predictor of molecular response for CML patients. The results demonstrate that IC $50^{\text {imatinib }}$ is a powerful pretreatment predictor [131]. The second factor is the activity of organic cation transporter 1 (OCT-1). There are two functions for OCT proteins, which are cellular uptake and excretion of a number of exogenous and endogenous cationic and uncharged substances. The OCT-1 protein activity (OA) can be measured by uptake in the presence and absence of a specific OCT1 inhibitor. It has been found that patients with high OA have a better molecular response than patients with low $\mathrm{OA}$; therefore, $\mathrm{OA}$ is considered a predictive factor for response to Imatinib, but not for Nilotinib or Dasatinib [131, 132]. White et al. propose [133] that, in CML patients treated with
Imatinib, the use of OA pretherapy was a predictor for longterm resistance risk and could be used to individualize dosage strategies. Thus, involving OA to estimate the response could lead to better results, but only for Imatinib therapy. A recent study has investigated the possible association between molecular response and a number of factors such as Sokal score, age, sex, and Imatinib dose [134]. It was also found that being female is a strong predictor [134]. A recent review of biomarkers that determine prognosis in CML also presented a list of prognostic indicators at diagnosis, such as the three scoring systems, BCR-ABL1 transcript type, and OA [135]. Another factor is the $B C R-A B L$ transcript type; CML patients with the b3a2 $B C R-A B L 1$ transcript type, compared to those with the b2a2 transcript type, demonstrate greater survival rates, while CML patients with the p190 transcript type are classified as high risk $[136,137]$.

In practice, clinicians aim to treat individual $\mathrm{CML}$ patients with the most beneficial therapy. This can be made possible by using accurate risk assessment methods at diagnosis. When there is any doubt about either the diagnosis or the recommended treatment, a second opinion is often sought before considering any treatment. The need for multiple prognostic scores can occur frequently in a complex problem that has multiple independent experts with varying expertise. When developing prognostic scores have different patient populations, each score can capture different knowledge. There are two general major objectives for combining prognostic scores: first, one prognostic score enhances the decision of another one; and second, it increases the reliability of the final decision. However, integrating multiple prognostic scores could generate conflict in decisions and may not be sufficient to make a final decision.

It is important that clinicians are comfortable with a wide range of prognostic scores that will help to identify risk category because a conflict between scores may be observed in some patients. Consistency is defined as a score that does 


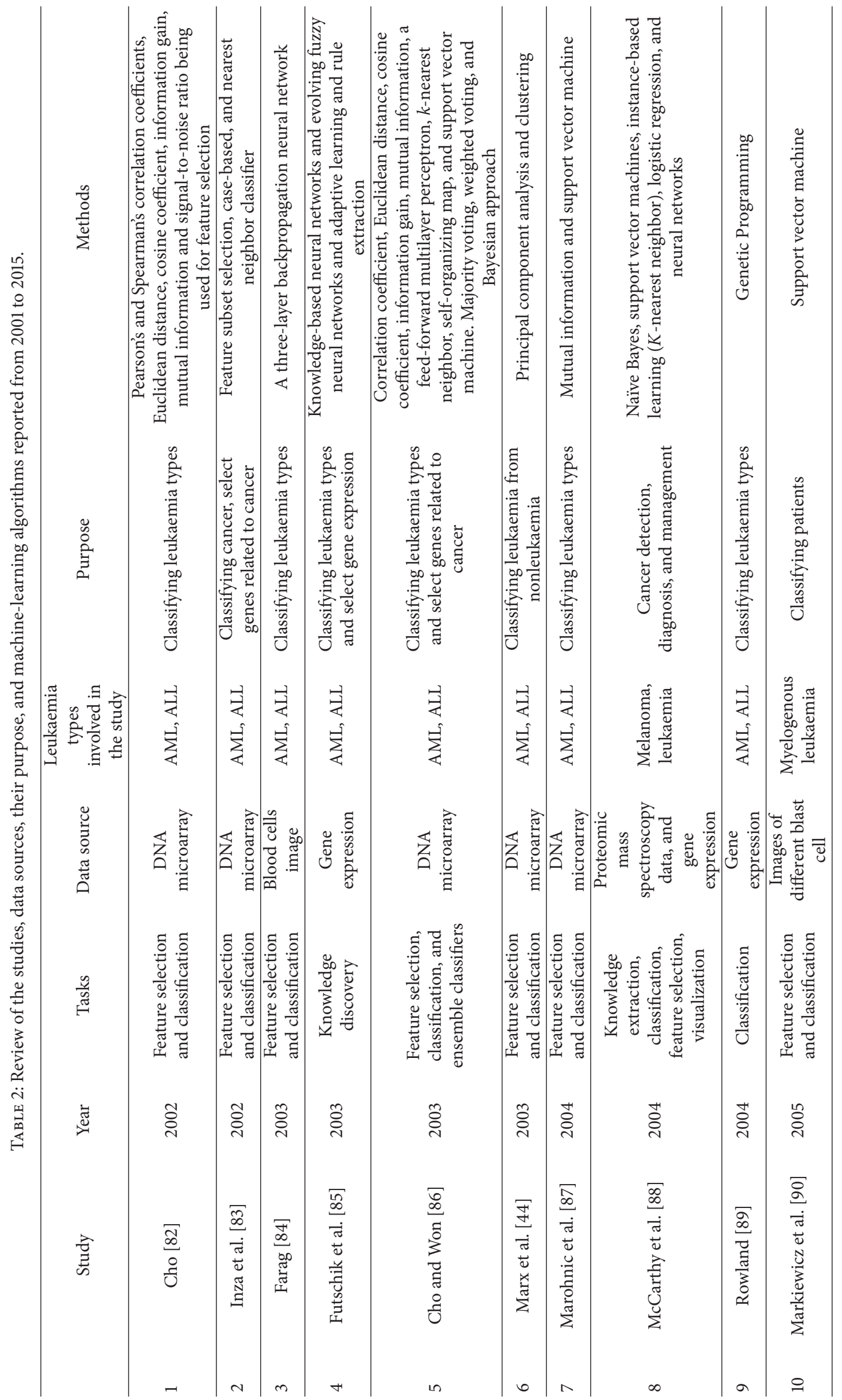









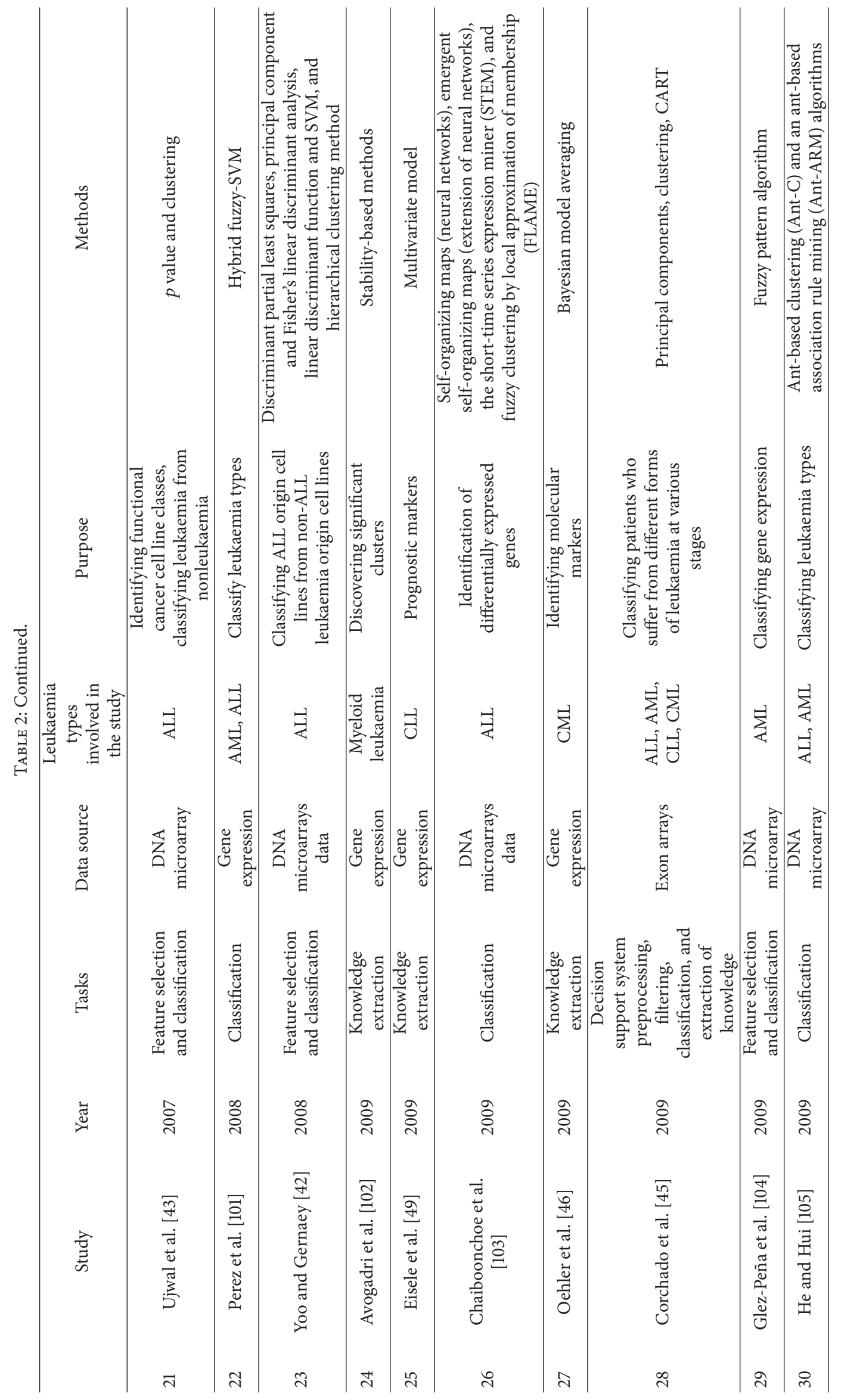




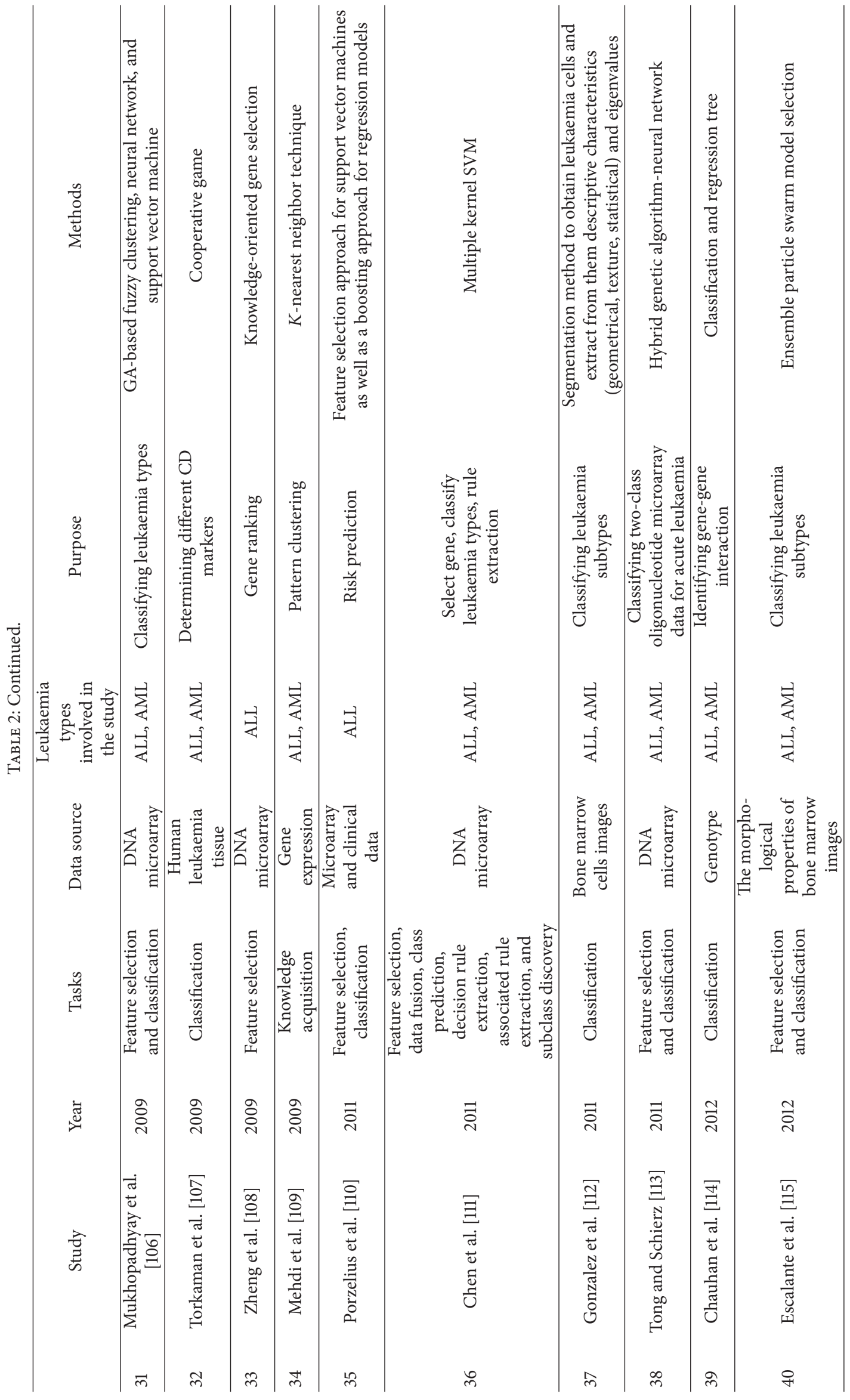




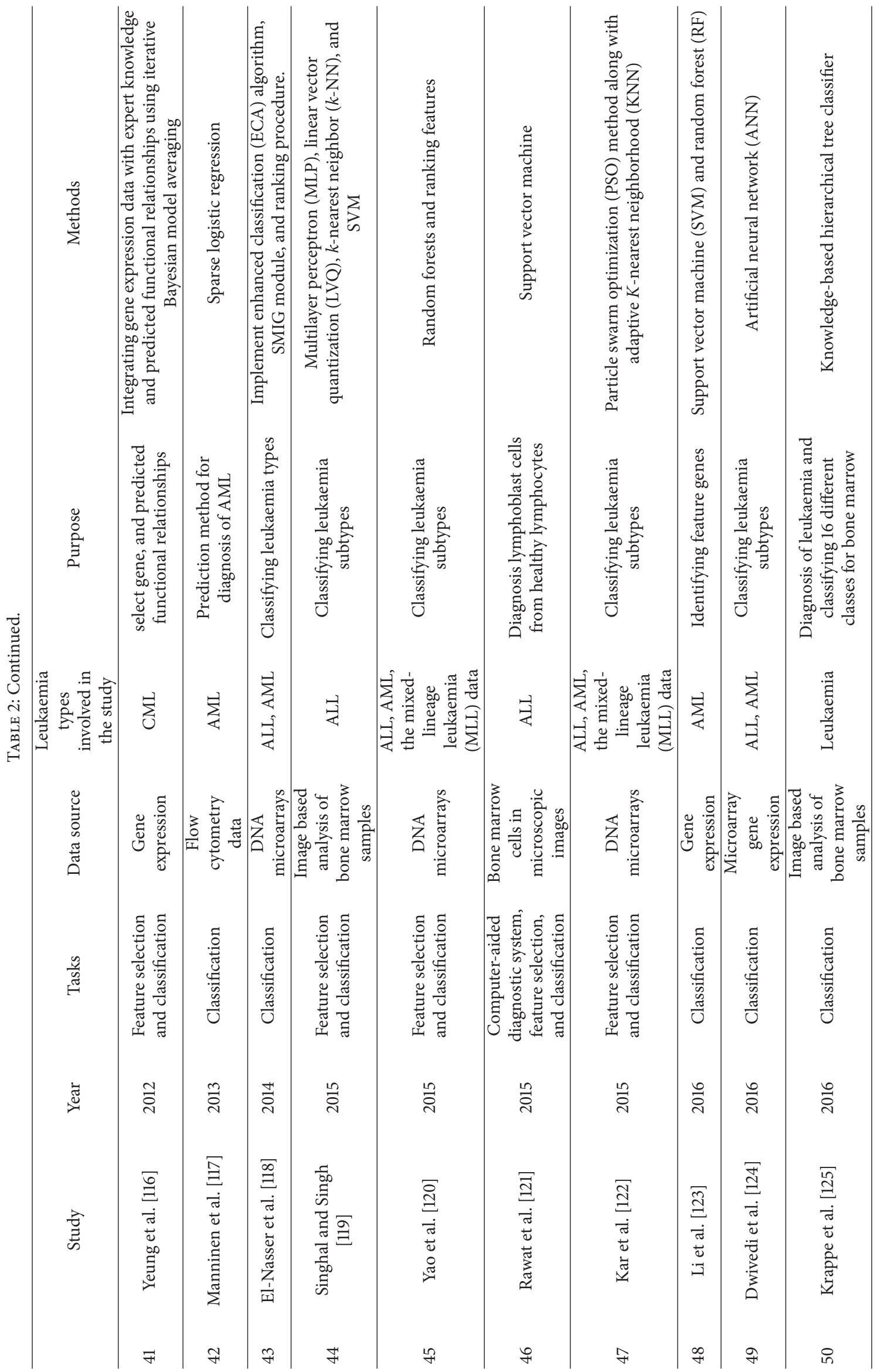




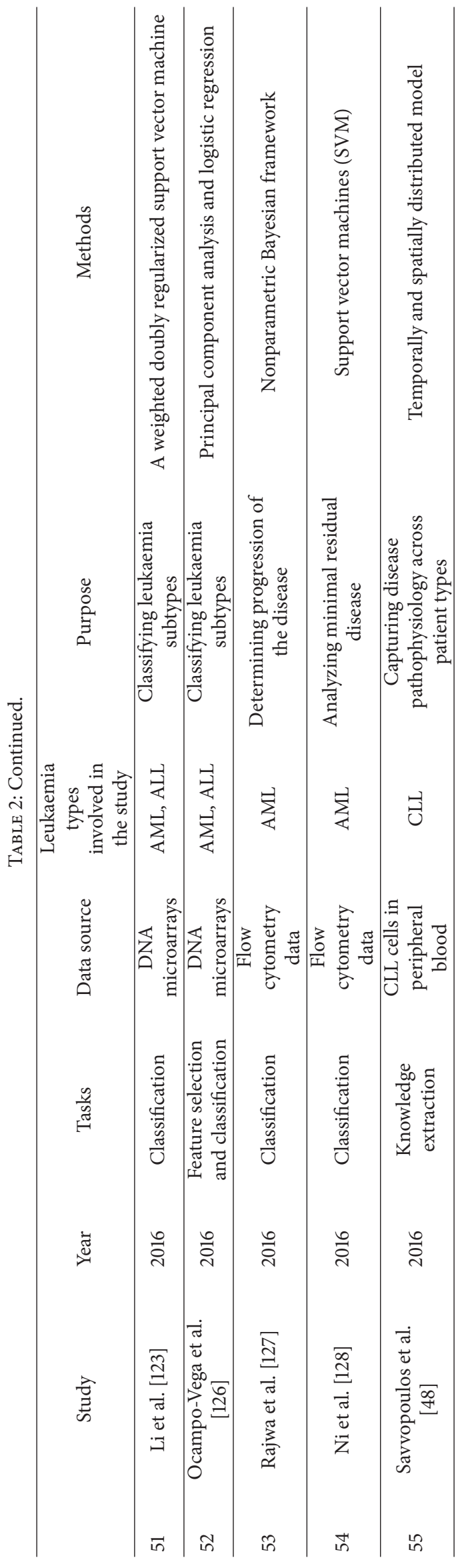


not contradict other prognostic scores. Consistency among prognostic scores can increase clinicians' trust, as they rely on such results to make appropriate treatment decisions. It is important to study and understand the consistency of scores to help clinicians categorize patients into suitable risk groups and subsequently make better therapeutic decisions.

In light of the aforementioned aspects, it is necessary to conduct a study that can contribute to the CML medical field by solving the previous issues. Using machine-learning techniques and fusion techniques to address these problems could produce promising results. The first proposed solution is to build a personalized medicine support system as a predictive model to combine strong molecular, clinical data, and predictive assays for CML patients that could probably predict an individual molecular response. Moreover, predicting an individual response leads to predicting warning groups for each TKI. From a computer-science perspective, the above issues could be resolved by using a machine-learning algorithm that combines the most effective predictive indicators to predict the outcomes for each TKI, based on existing clinical profiles for individual CML patient characteristics. The main goal of this review is to improve the ability to manage CML disease in individual CML patients. Therefore, CML is an example of a research opportunity to predict the molecular response to TKI treatment. Using intelligent computing techniques could bring about promising results for CML patients.

3.5. Based on the Task. Most of the studies that used machine learning and data mining incorporated two major tasks: feature selection and classification (Figure 5). Although dissimilar feature-selection algorithms may possibly choose dissimilar pertinent genes or diverse numbers of relevant genes or bring about different levels of classification precision [138], feature selection can utilize observations and functions to obtain dimensions for exploring optimal solutions [139]. In addition, selection of a subset in a classifier reduces the computational time and costs of study, thereby increasing classification accuracy.

Many studies [53, 89, 91, 103, 105, 112, 118] used classification algorithms without feature-selection techniques. Since cancer tumors are highly diverse in their genetic patterns and progressions, DNA arrays provide a platform to obtain the best measurements and observations, helping assign objectives to one relevant feature set and hence contributing to precise convergence toward optimal results [140]. However, some researchers $[42-44,82,86,87,94,96-99,104,108,113$, $115,122,141,142]$ applied two common methods in feature selection: filter and wrapper approaches with classification algorithms. Considering feature-selection techniques is an essential preprocessing method mandated for classification processes [103].

Knowledge extraction or acquisition has been a great challenge for researchers, as they exhibit unusual characteristics in many different genes relative to the number of tumor samples. AML acquires a similar appearance to ALL, which makes it nearly impossible for researchers to distinguish between synonymous patterns. However, Cho et al. [143] proposed an approach to form the optimal linear classifier

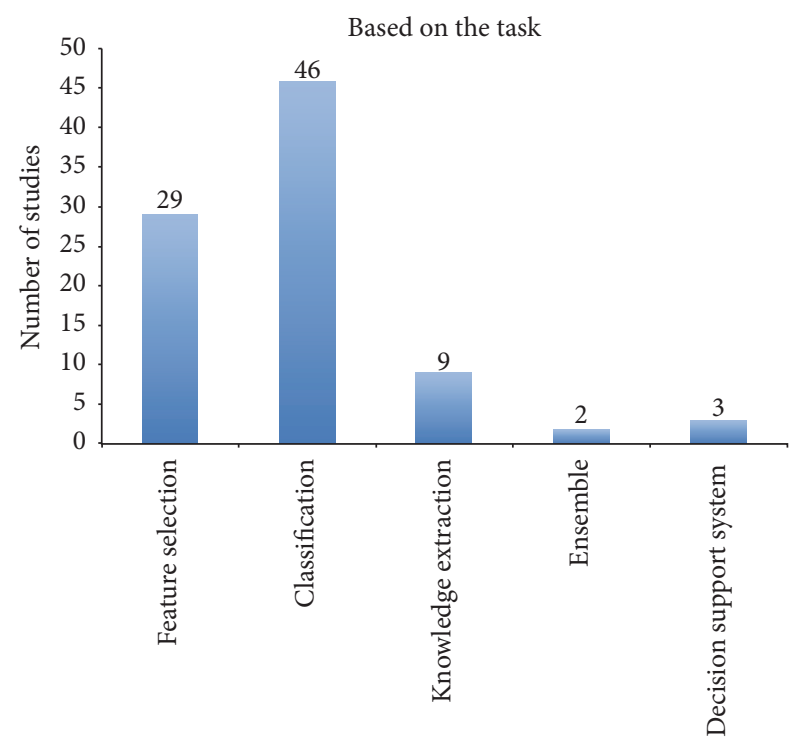

FIGURE 5: Summary of the frequency of studies based on the task.

by means of gene-expression data. They used discriminant partial least squares and linear discriminant analysis to differentiate between acute leukaemia subtypes. They found that these methods offered a satisfactory level of precision. They concluded that the suggested method builds the optimal classifier made up of a highly accurate, small-size predictor.

Using multiple algorithms for knowledge extraction and classification has not attracted much interest from leukaemia researchers in previous studies $[45,140,144]$. Cho and Won [86] were of the view that conventional machine learning is incapable of delivering accurate information. For this reason, they developed a novel ensemble machine-learning approach for microarray classification. Results indicated that the ensemble machine-learning approaches accuracy of almost $97 \%$ in leukaemia classification, which makes it a better alternative to basic machine-learning methods.

Among the 55 studies, three groups of researchers [45, $121,140]$ built decision support systems, whose subfunctions included multiple tasks. The first decision support system [45] was built to support leukaemia diagnosis using exon array analysis. The system combined intelligent techniques, such as preprocessing and data-filtering techniques, clustering for classifying patients, and extraction of knowledge techniques. The authors suggested that further study of bone marrow or blood samples may assist in diagnosis of leukaemia stages. The second study [140] was conducted to extract decision rules using a developed SVM. This study comprised carrying out multiple tasks, including data fusion, feature selection, making a prediction model based on gene-expression data, and knowledge extraction. The third study [121] involved developing decision support to identify unhealthy ALL cells using feature-selection techniques with SVM.

From the review of studies based on the task, the need for personalized medicine in CML results in multiple active TKI therapies as molecular targeted therapy available for CML, multiple strategies utilized for frontline CML therapy, 
heterogeneity in responses, and multiple prognostic scores and predictive assays.

Therapy takes the form of two major strategies: (i) frontline Imatinib or (ii) frontline second-generation TKIs such as Nilotinib or Dasatinib [4]. Despite the remarkable increase in the survival of CML patients treated with Imatinib, some patients discontinue Imatinib therapy due to intolerance, resistance, or progression. In the IRIS [64] trial, it is demonstrated that variations in molecular response at 12 and 18 months of Imatinib was due to, in about $40 \%$ of cases, discontinuing Imatinib because of intolerance or resistance and due to further progression observed in $7 \%$ of CML patients.

Hematologic, cytogenetic, and molecular strategies for monitoring patient responses to therapies are used by European LeukaemiaNet [145]. To monitor molecular response, RQ-PCR, which is a sensitive technique, is used to quantify the level of $B C R-A B L 1$ mRNA transcripts in the peripheral blood of patients. Molecular monitoring is considered to be a standard guide to clinical management in CML $[146,147]$. The prediction of long-term molecular response to frontline Imatinib in CML can help clinicians to select the best treatment protocols for CML patients. Patients predicted not to achieve MMR in the long term might be better treated with alternative frontline therapies, such as Nilotinib or Dasatinib. Opportunities to improve individual care for CML patients exist in the appropriate prediction of variation in treatment response to support physicians in treatment decisions.

Prognostic scores are used to personalize CML patient care by predicting responses to therapy. Although the prognostic scores (Sokal, Hasford, EUTOS, and the ELTS scores) remain in use today, they were developed either for identifying risk groups or for predicting cytogenetic response to therapy, but not for molecular response. Although two predictive assays, IC50 ${ }^{\text {imatinib }}$ and OCT-1 activity (OA), were developed to predict molecular response, according to current knowledge, a model using assays to predict molecular response has not previously been considered. The combination of predictive assays results in greater predictive power than that which each predictor provides alone $[148,149]$.

\section{Conclusion}

Modern oncology is experiencing a paradigm shift toward personalized medicine, which aims to direct medical agents toward the tumor site. The field of molecular medicine is also undergoing transformational changes that are bringing a much needed revolution in healthcare. This breakthrough was made possible by technologies in genetic studies that led to the sequencing of the human genome. An analysis of biological samples from whole organisms has now been made possible. In addition, this invention has given a new lease of life to the treatment of cancer. However, the majority of cancer patients have been shown to develop adverse drug reactions due to overreliance on certain medications.

Intelligent techniques may be useful for clinicians in decision-making, warning of specific problems or providing treatment recommendations [150]. In that regard, it would be worthwhile building personalized medicine support system to work as predictive models that integrate molecularbased data to predict cancer susceptibility, including risk assessment, prediction of the probability of developing a type of cancer prior to occurrence of the disease, prediction of recurring cancer, and the prediction of cancer outcomes, such as survivability, life expectancy, and response to therapy or progression. This is highly advantageous since only a quarter of cancer patients respond positively to the drugs prescribed to them. Therefore, it is important to investigate the current development of using molecular information in intelligent models for personalized medicine.

The use of personalized medicine support systems in medicine will bring a ray of hope to the treatment of leukaemia. Other frontiers of personalized medicine research, such as the role of genetics in infectious diseases, proteomics, epigenetics, and metabolomics, were not covered by this review and are out of scope of this research. This review was conducted based on current developments of personalized medicine support systems, and a systematized literature review was carried out on intelligent techniques using molecular data analysis in leukaemia. Both sets of literature led to identifying opportunities for further research for personalized medicine support systems in one category of leukaemia, namely, chronic myeloid leukaemia. We speculate that this paper will assist health informatics and biomedical and bioinformatics in order to answer specific technical questions to help develop future research into leukaemia from a technical perspective.

\section{Disclosure}

The funding agreement ensured the authors' independence in publishing the report.

\section{Conflicts of Interest}

The authors declare that they have no conflicts of interest.

\section{Authors' Contributions}

Haneen Banjar designed and performed the research, analyzed data, and wrote the manuscript. All the listed authors contributed substantially to drafts and revisions to the manuscript and approved the current revised version.

\section{Acknowledgments}

Financial support for this study was provided in part by a grant from King Abdulaziz University.

\section{References}

[1] K. K. Jain, Basics of Personalized Medicine Textbook of Personalized Medicine, Springer, New York, NY, USA, 2009.

[2] S. J. Swierzewski, Leukemia Basics: Remedy Health Media; 1999, http://www.healthcommunities.com/leukemia/types.shtml. 
[3] G. S. Ginsburg and H. F. Willard, "Genomic and personalized medicine: foundations and applications," Translational Research, vol. 154, no. 6, pp. 277-287, 2009.

[4] J. Cortes and H. Kantarjian, "How I treat newly diagnosed chronic phase CML," Blood, vol. 120, no. 7, pp. 1390-1397, 2012.

[5] I. Kouris, C. Tsirmpas, S. G. Mougiakakou, D. Iliopoulou, and D. Koutsouris, "E-health towards ecumenical framework for personalized medicine via Decision Support System," in Proceedings of the 2010 32nd Annual International Conference of the IEEE Engineering in Medicine and Biology Society, EMBC'10, pp. 2881-2885, September 2010.

[6] C. L. Overby, P. Tarczy-Hornoch, I. J. Kalet et al., "Developing a prototype system for integrating pharmacogenomics findings into clinical practice," Journal of Personalized Medicine, vol. 2, no. 4, pp. 241-256, 2012.

[7] N. Douali and M.-C. Jaulent, "Genomic and personalized medicine decision support system," in Proceedings of the 1st International Conference on Complex Systems, ICCS 2012, November 2012.

[8] S. G. Mougiakakou, I. K. Valavanis, G. Karkalis, S. Marinos, K. A. Grimaldi, and K. S. Nikita, "An integrated web-based platform for the provision of personalized advice in people at high risk for CVD," in Proceedings of the 9th International Conference on Information Technology and Applications in Biomedicine, ITAB 2009, November 2009.

[9] D. C. Klonoff, "Personalized medicine for diabetes," Journal of Diabetes Science and Technology, vol. 2, no. 3, pp. 335-341, 2008.

[10] K. Zarkogianni, S. G. Mougiakakou, A. Prountzou, A. Vazeou, C. S. Bartsocas, and K. S. Nikita, "An insulin infusion advisory system for type 1 diabetes patients based on non-linear model predictive control methods," in Proceedings of the Engineering in Medicine and Biology Society, 2007 EMBS 2007 29th Annual International Conference of the IEEE, pp. 22-26, 2007.

[11] Y. Goletsis, T. P. Exarchos, N. Giannakeas, and D. I. Fotiadis, "Intelligent patient profiling for diagnosis, staging and treatment selection in colon cancer," in Proceedings of the 8th IEEE International Conference on BioInformatics and BioEngineering, BIBE 2008, October 2008.

[12] A. Kulkarni, B. S. C. Naveen Kumar, V. Ravi, and U. S. Murthy, "Colon cancer prediction with genetics profiles using evolutionary techniques," Expert Systems with Applications, vol. 38, no. 3, pp. 2752-2757, 2011.

[13] H. Moon, H. Ahn, R. L. Kodell, S. Baek, C.-J. Lin, and J. J. Chen, "Ensemble methods for classification of patients for personalized medicine with high-dimensional data," Artificial Intelligence in Medicine, vol. 41, no. 3, pp. 197-207, 2007.

[14] V. J. Weigman Jr., High Level Integration of Genomic Data for Improving Prediction, Prognostication and Classification of Breast Tumors. Proquest Dissertations and Theses, University of North Carolina, Chapel Hill, NC, USA, 2010.

[15] M. Ture, F. Tokatli, and I. Kurt Omurlu, "The comparisons of prognostic indexes using data mining techniques and Cox regression analysis in the breast cancer data," Expert Systems with Applications, vol. 36, no. 4, pp. 8247-8254, 2009.

[16] M. U. Khan, J. P. Choi, H. Shin, and M. Kim, "Predicting breast cancer survivability using fuzzy decision trees for personalized healthcare," in Proceedings of the 30th Annual International Conference of the IEEE Engineering in Medicine and Biology Society, EMBS'08, pp. 5148-5151, August 2008.

[17] S. Lekkas and L. Mikhailov, Breast Cancer Diagnosis Based on Evolvable Fuzzy Classifiers and Feature Selection. Applications and Innovations in Intelligent Systems XVI, Springer, London, UK, 2009.

[18] D. Lavanya and K. Usha Rani, "Ensemble Decision Making System for Breast Cancer Data," International Journal of Computer Applications, vol. 51, no. 17, pp. 19-23, 2012.

[19] D. Lavanya and KU. Raniv, "Analysis of feature selection with classfication: breast cancer datasets," Indian Journal of Computer Science and Engineering (IJCSE), vol. 2, no. 5, p. 763, 2011.

[20] A. Keleş, A. Keleş, and U. Yavuz, "Expert system based on neuro-fuzzy rules for diagnosis breast cancer," Expert Systems with Applications, vol. 38, no. 5, pp. 5719-5726, 2011.

[21] B. Jahn, N. Muhlberger, J. Wurm, and U. Siebert, "Decisionanalytic models for breast cancer: Do currently published models meet the requirements of personalized medicine?" in Proceedings of the 2008 Winter Simulation Conference (WSC), pp. 2946-2946, Miami, FL, USA, December 2008.

[22] J. M. Garibaldi, S.-M. Zhou, X.-Y. Wang, R. I. John, and I. O. Ellis, "Incorporation of expert variability into breast cancer treatment recommendation in designing clinical protocol guided fuzzy rule system models," Journal of Biomedical Informatics, vol. 45, no. 3, pp. 447-459, 2012.

[23] D. Delen, G. Walker, and A. Kadam, "Predicting breast cancer survivability: a comparison of three data mining methods," Artificial Intelligence in Medicine, vol. 34, no. 2, pp. 113-127, 2005.

[24] C. Chen, S.-R. Sun, Y.-P. Gong et al., "Quantum dots-based molecular classification of breast cancer by quantitative spectroanalysis of hormone receptors and HER2," Biomaterials, vol. 32, no. 30, pp. 7592-7599, 2011.

[25] M. Arora and D. Tagra, "Neuro-fuzzy expert system for breast cancer diagnosis," in Proceedings of the 2012 International Conference on Advances in Computing, Communications and Informatics, ICACCI 2012, pp. 979-985, August 2012.

[26] A. Ali, A. Tufail, U. Khan, and M. Kim, "A survey of prediction models for breast cancer survivability," in Proceedings of the 2nd International Conference on Interaction Sciences: Information Technology, Culture and Human, ICIS 2009, pp. 1259-1262, November 2009.

[27] A. Ali, U. Khan, A. Tufail, and M. Kim, "Analyzing potential of SVM based classifiers for intelligent and less invasive breast cancer prognosis," in Proceedings of the 2nd International Conference on Computer Engineering and Applications, ICCEA 2010, pp. 313-319, March 2010.

[28] E. A. Rakha, D. Soria, A. R. Green et al., "Nottingham prognostic index plus (NPI+): A modern clinical decision making tool in breast cancer," British Journal of Cancer, vol. 110, no. 7, pp. 1688-1697, 2014.

[29] K. Nagasaki and Y. Miki, "Molecular prediction of the therapeutic response to neoadjuvant chemotherapy in breast cancer," Breast Cancer, vol. 15, no. 2, pp. 117-120, 2008.

[30] T. Kempowsky-Hamon, C. Valle, M. Lacroix-Triki et al., "Fuzzy logic selection as a new reliable tool to identify molecular grade signatures in breast cancer-the INNODIAG study," BMC Medical Genomics, vol. 8, no. 1, article 3, 2015.

[31] Arsene CTC, Lisboa PJG. Chapter 8 - Artificial Neural Networks Used in the Survival Analysis of Breast Cancer Patients: A Node-Negative Study. In: Outcome Prediction in Cancer [Internet]. Amsterdam: Elsevier; [191-239], http://www .sciencedirect.com/science/article/pii/B9780444528551500106.

[32] C. Voichita, Towards Personalized Medicine Using Systems Biology and Machine Learning, Wayne State University Dissertations, Detroit, MI, USA, 2013. 
[33] D.-C. Kim, X. Wang, C.-R. Yang, and J. X. Gao, "A framework for personalized medicine: prediction of drug sensitivity in cancer by proteomic profiling," Proteome Science, vol. 10, no. 1, 2012.

[34] D.-C. Kim, J. Gao, X. Wang, and C.-R. Yang, "A framework for personalized medicine with reverse phase protein array and drug sensitivity," in Proceedings of the 2011 IEEE International Conference on Bioinformatics and Biomedicine, BIBM 2011, pp. 426-429, November 2011.

[35] P. K. Anooj, "Clinical decision support system: risk level prediction of heart disease using weighted fuzzy rules," Journal of King Saud University-Computer and Information Sciences, vol. 24, no. 1, pp. 27-40, 2012.

[36] A. M. Honka, M. J. van Gils, and J. Parkka, "A personalized approach for predicting the effect of aerobic exercise on blood pressure using a fuzzy inference system," in Proceedings of the Annual International Conference of the IEEE Engineering in Medicine and Biology Society (EMBC '11), pp. 8299-8302, Boston, Mass, USA, August 2011.

[37] H. Michael and M. Crawford, "Individualized medicine by biomarkers," Clinical Cardiology Alert, vol. 302, pp. 49-57, 2009.

[38] B. S. Shastry, "Pharmacogenetics and the concept of individualized medicine," Pharmacogenomics Journal, vol. 6, no. 1, pp. 16-21, 2006.

[39] GLIVEC Tablets - Summary of Product Characteristics (SPC): electronic Medicines Compendium; 2001, http://www .medicines.org.uk/emc/medicine/15014/SPC/GLIVEC+Tablets/.

[40] M. J. Grant and A. Booth, "A typology of reviews: an analysis of 14 review types and associated methodologies," Health Information \& Libraries Journal, vol. 26, no. 2, pp. 91-108, 2009.

[41] Cancer Program Legacy Publication Resources, http://www .broadinstitute.org/cgi-bin/cancer/datasets.cgi.

[42] C. K. Yoo and K. V. Gernaey, "Classification and diagnostic output prediction of cancer using gene expression profiling and supervised machine learning algorithms," Journal of Chemical Engineering of Japan, vol. 41, no. 9, pp. 898-914, 2008.

[43] M. L. Ujwal, P. Hoffman, and K. A. Marx, "A machine learning approach to pharmacological profiling of the quinone scaffold in the NCI database: A compound class enriched in those effective against melanoma and leukemia cell lines," in Proceedings of the 7th IEEE International Conference on Bioinformatics and Bioengineering, BIBE, pp. 456-463, January 2007.

[44] K. A. Marx, P. O'Neil, P. Hoffman, and M. L. Ujwal, "Data Mining the NCI Cancer Cell Line Compound GI50 Values: Identifying Quinone Subtypes Effective Against Melanoma and Leukemia Cell Classes," Journal of Chemical Information and Computer Sciences, vol. 43, no. 5, pp. 1652-1667, 2003.

[45] J. M. Corchado, J. F. de Paz, S. Rodríguez, and J. Bajo, "Model of experts for decision support in the diagnosis of leukemia patients," Artificial Intelligence in Medicine, vol. 46, no. 3, pp. 179-200, 2009.

[46] V. G. Oehler, Y. Y. Ka, Y. E. Choi, R. E. Bumgarner, A. E. Raftery, and J. P. Radich, "The derivation of diagnostic markers of chronic myeloid leukemia progression from microarray data," Blood, vol. 114, no. 15, pp. 3292-3298, 2009.

[47] K. Y. Yeung, T. A. Gooley, A. Zhang, A. E. Raftery, J. P. Radich, and V. G. Oehler, "Predicting relapse prior to transplantation in chronic myeloid leukemia by integrating expert knowledge and expression data," Bioinformatics, vol. 28, no. 6, Article ID bts059, pp. 823-830, 2012.

[48] S. Savvopoulos, R. Misener, N. Panoskaltsis, E. N. Pistikopoulos, and A. Mantalaris, "A Personalized Framework for Dynamic
Modeling of Disease Trajectories in Chronic Lymphocytic Leukemia," IEEE Transactions on Biomedical Engineering, vol. 63, no. 11, pp. 2396-2404, 2016.

[49] L. Eisele, R. Prinz, L. Klein-Hitpass et al., "Combined PER2 and CRY1 expression predicts outcome in chronic lymphocytic leukemia," European Journal of Haematology, vol. 83, no. 4, pp. 320-327, 2009.

[50] Leukaemia in Australia: The Australian Institute of Health and Welfare, http://www.aihw.gov.au/cancer/leukaemia/.

[51] J. F. Apperley, "Part I: mechanisms of resistance to imatinib in chronic myeloid leukaemia," The Lancet Oncology, vol. 8, no. 11, pp. 1018-1029, 2007.

[52] D. L. White and T. P. Hughes, "Predicting the response of CML patients to tyrosine kinase inhibitor therapy," Current Hematologic Malignancy Reports, vol. 6, no. 2, pp. 88-95, 2011.

[53] A. Torkaman, N. M. Charkari, and M. Aghaeipour, "A new classification approach based on cooperative game," in Proceedings of the 2009 14th International CSI Computer Conference, CSICC 2009, pp. 458-463, October 2009.

[54] B. Welch and K. Kawamoto, "The Need for Clinical Decision Support Integrated with the Electronic Health Record for the Clinical Application of Whole Genome Sequencing Information," Journal of Personalized Medicine, vol. 3, no. 4, pp. 306-325, 2013.

[55] J. C. Weiss, S. Natarajan, P. L. Peissig, C. A. McCarty, and D. Page, "Machine learning for personalized medicine: Predicting primary myocardial infarction from electronic health records," AI Magazine, vol. 33, no. 4, pp. 33-45, 2012.

[56] CL. Overby, A Clinical Decision Support Model for Incorporating Pharmacogenomics Knowledge Into Electronic Health Records for Drug Therapy Individualization: A Microcosm of Personalized Medicine, University of Washington, Seattle, WA, USA, 2011.

[57] B. Hirsch and A. Abernethy, "Structured Decision-Making: Using Personalized Medicine to Improve the Value of Cancer Care," Journal of Personalized Medicine, vol. 3, no. 1, pp. 1-13, 2013.

[58] H. Yu, G. Gu, H. Liu, and J. Shen, "A framework for microarray data-based tumor diagnostic system with improving performance incrementally," Expert Systems with Applications, vol. 37, no. 9, pp. 6682-6688, 2010.

[59] Global Alliance for Genomics and Health, http://genomicsandhealth.org.

[60] National Institutes of Health, U.S.: Turning Discovery Into Health, https://www.nih.gov.

[61] M. Kwiatkowska, A. S. Atkins, N. T. Ayas, and C. F. Ryan, "Integrating knowledge-driven and data-driven approaches for the derivation of clinical prediction rules," in Proceedings of the ICMLA 2005: 4th International Conference on Machine Learning and Applications, pp. 171-176, December 2005.

[62] S. Saussele and M. Pfirrmann, "Clinical trials in chronic myeloid leukemia," Current Hematologic Malignancy Reports, vol. 7, no. 2, pp. 109-115, 2012.

[63] M. Baccarani, F. Guilhot, R. A. Larson, S. G. O’Brien, and B. J. Druker, "Outcomes by cytogenetic and molecular response at 12 and 18 months of imatinib in patients with newly diagnosed chronic myeloid leukemia (CML) in chronic phase (CP) in the IRIS tria," in Proceedings of the The American Society of Hematology 48th Annual Meeting and Exposition, vol. 2006, Orlando, FL, USA.

[64] M. Deininger, SG. O’Brien, F. Guilhot et al., "International Randomized Study of Interferon Vs STI571 (IRIS) 8-Year 
Follow up: Sustained Survival and Low Risk for Progression or Events in Patients with Newly Diagnosed Chronic Myeloid Leukemia in Chronic Phase (CML-CP) Treated with Imatinib," in Proceedings of the Blood (ASH Annual Meeting Abstracts), 2009.

[65] W. Alexander and T. P. Hughes, "American Society of Hematology, 52nd Annual Meeting and Exposition: Nilotinib (Tasigna) found superior to imatinib (Gleevec) in chronic-phase chronic myeloid leukemia: ENESTnd update," $P$ and T, vol. 36, no. 2, pp. 100-101, 2011.

[66] H. Kantarjian, N. P. Shah, A. Hochhaus et al., "Dasatinib versus imatinib in newly diagnosed chronic-phase chronic myeloid leukemia," The New England Journal of Medicine, vol. 362, no. 24, pp. 2260-2270, 2010.

[67] T. P. Hughes, A. Hochhaus, G. Saglio et al., "ENESTnd Update: Continued Superiority of Nilotinib Versus Imatinib In Patients with Newly Diagnosed Chronic Myeloid Leukemia In Chronic Phase (CML-CP)," in Proceedings of the Blood (ASH Annual Meeting Abstracts), 2010.

[68] P. L. Alvey, N. J. Preston, and M. F. Greaves, "High performance for expert systems: II. A system for leukaemia diagnosis," Informatics for Health and Social Care, vol. 12, no. 2, pp. 97-114, 1987.

[69] Y. M. Chae, Q. Park, K. S. Park, and M. Young, "Development of medical decision support system for leukemia management," Expert Systems with Applications, vol. 15, no. 3-4, pp. 309-315, 1998.

[70] J. Hasford, M. Pfirrmann, R. Hehlmann et al., "Prognosis and prognostic factors for patients with chronic myeloid leukemia: Nontransplant therapy,' Seminars in Hematology, vol. 40, no. 1, pp. 4-12, 2003.

[71] N. Mašić, A. Gagro, S. Rabatić et al., "Decision-tree approach to the immunophenotype-based prognosis of the B- cell chronic lymphocytic leukemia," American Journal of Hematology, vol. 59, no. 2, pp. 143-148, 1998.

[72] J. Hasford, M. Baccarani, V. Hoffmann et al., "Predicting complete cytogenetic response and subsequent progressionfree survival in 2060 patients with CML on imatinib treatment: the EUTOS score," Blood, vol. 114, no. 22, pp. 686-692, 2009.

[73] M. Breccia, F. Stagno, A. Gozzini et al., "Hammersmith score application identifies chronic myeloid leukemia patients with poor prognosis before treatment with second-generation tyrosine kinase inhibitors," American Journal of Hematology, vol. 116, no. 21, pp. 1395-1396, 2010.

[74] C. N. A. M. Oldenhuis, S. F. Oosting, J. A. Gietema, and E. G. E. de Vries, "Prognostic versus predictive value of biomarkers in oncology," European Journal of Cancer, vol. 44, no. 7, pp. 946953, 2008.

[75] M. Agrawal, R. J. Garg, H. Kantarjian, and J. Cortes, "Chronic myeloid leukemia in the tyrosine kinase inhibitor era: What is the "best" therapy?" Current Oncology Reports, vol. 12, no. 5, pp. 302-313, 2010.

[76] F. Guilhot and J. Guilhot, "Predicting response in CML," Blood, vol. 117, no. 6, pp. 1773-1774, 2011.

[77] E. Jabbour, H. Kantarjian, S. O’Brien et al., "Predictive factors for outcome and response in patients treated with secondgeneration tyrosine kinase inhibitors for chronic myeloid leukemia in chronic phase after imatinib failure," Blood, vol. 117, no. 6, pp. 1822-1827, 2011.

[78] J. Sokal, E. Cox, M. Baccarani, and S. Tura, "Prognostic discrimination in good-risk chronic granulocytic leukemia," Blood, vol. 63, pp. 789-799, 1984.
[79] J. Hasford, M. Pfirrmann, R. Hehlmann et al., "A new prognostic score for survival of patients with chronic myeloid leukemia treated with interferon alfa. Writing Committee for the Collaborative CML Prognostic Factors Project Group," Journal of the National Cancer Institute, vol. 90, no. 11, pp. 850-858, 1998.

[80] J. Hasford, M. Baccarani, V. Hoffmann et al., "Predicting complete cytogenetic response and subsequent progressionfree survival in 2060 patients with CML on imatinib treatment: the EUTOS score," Blood, vol. 118, no. 3, pp. 686-692, 2011.

[81] V.S. Hoffmann, M. Baccarani, D. Lindoerfer et al., "The EUTOS prognostic score: review and validation in 1288 patients with CML treated frontline with imatinib," Leukemia, vol. 27, no. 10, pp. 2016-2022, 2013.

[82] S.-B. Cho, "Exploring features and classifiers to classify gene expression profiles of acute leukemia," International Journal of Pattern Recognition and Artificial Intelligence, vol. 16, no. 7, pp. 831-844, 2002.

[83] I. Inza, B. Sierra, R. Blanco, and P. Larrañaga, "Gene selection by sequential search wrapper approaches in microarray cancer class prediction," Journal of Intelligent \& Fuzzy Systems, vol. 12, no. 1, pp. 25-33, 2002.

[84] A. Farag, "Computer Based Acute Leukemia Classification," in Proceedings of the 2003 46th Midwest Symposium on Circuits and Systems, pp. 701-703, Cairo, Egypt.

[85] M. E. Futschik, A. Reeve, and N. Kasabov, "Evolving connectionist systems for knowledge discovery from gene expression data of cancer tissue," Artificial Intelligence in Medicine, vol. 28, no. 2, pp. 165-189, 2003.

[86] S.-B. Cho and H.-H. Won, "Data mining for GENE expression profiles from DNA microarray," International Journal of Software Engineering and Knowledge Engineering, vol. 13, no. 6, pp. 593-608, 2003.

[87] V. Marohnic, Z. Debeljak, and N. Bogunovic, "Mutual information based reduction of data mining dimensionality in gene expression analysis. Information Technology Interfaces," in Proceedings of the 26th International Conference, pp. 7-10, 2004.

[88] J. F. McCarthy, K. A. Marx, P. E. Hoffman et al., "Applications of machine learning and high-dimensional visualization in cancer detection, diagnosis, and management," Annals of the New York Academy of Sciences, vol. 1020, pp. 239-262, 2004.

[89] J. Rowland, "On genetic programming and knowledge discovery in transcriptome data," in Proceedings of the 2004 Congress on Evolutionary Computation, pp. 158-165, Portland, OR, USA.

[90] T. Markiewicz, S. Osowski, B. Marianska, and L. Moszczyński, "Automatic recognition of the blood cells of myelogenous leukemia using SVM," in Proceedings of the International Joint Conference on Neural Networks, IJCNN 2005, pp. 2496-2501, August 2005.

[91] W. L. Tung and C. Quek, "GenSo-FDSS: a neural-fuzzy decision support system for pediatric ALL cancer subtype identification using gene expression data," Artificial Intelligence in Medicine, vol. 33, no. 1, pp. 61-88, 2005.

[92] H. Nguyen, S. Ohn, J. Park, and K. Park, "Combined Kernel Function Approach in SVM for Diagnosis of Cancer," in Advances in Natural Computation, vol. 3610 of Lecture Notes in Computer Science, pp. 1017-1026, Springer, Berlin, Germany, 2005.

[93] V. P. Plagianakos, D. K. Tasoulis, and M. N. Vrahatis, "Computational intelligence techniques for acute leukemia gene expression data classification," in Proceedings of the International Joint 
Conference on Neural Networks, IJCNN 2005, pp. 2469-2474, August 2005.

[94] F. Li and Y. Yang, "Analysis of recursive gene selection approaches from microarray data," Bioinformatics, vol. 21, no. 19, pp. 3741-3747, 2005.

[95] W. Jinlian, L. Jiangeng, and R. Xiaogang, "Mining leukemia gene association structure with DNA microarray," in Proceedings of the 2005 International Conference on Neural Networks and Brain Proceedings, ICNNB'05, pp. 695-701, October 2005.

[96] F. Diaz, F. Fdez-Riverola, D. Glez-Pena, and J. M. Corchado, "Using fuzzy patterns for gene selection and data reduction on microarray data," in Intelligent Data Engineering and Automated Learning - Ideal, E. Corchado, H. Yin, V. Botti, and C. Fyfe, Eds., Lecture Notes in Computer Science, pp. 1087-94, 2006.

[97] C. Feng and W. Lipo, "A Novel Approach Searching for Discriminative Gene Sets. Systems, Man and Cybernetics," in Proceeding of the 2006 SMC '06 IEEE International Conference, pp. 8-11, 2006.

[98] H. Nguyen and S. Ohn, "DRFE: Dynamic Recursive Feature Elimination for Gene Identification Based on Random Forest," in Neural Information Processing, vol. 4234 of Lecture Notes in Computer Science, pp. 1-10, Springer, Berlin, Germany, 2006.

[99] W. Shulin, C. Huowang, W. Ji, and Z. Dingxing, "Molecular Diagnosis of Tumor Based on Independent Component Analysis and Support Vector Machines. Computational Intelligence and Security, 2006 International Conference on," in Proceedings of the Computational Intelligence and Security, 2006 International Conference, 2006.

[100] Y. Chen, M. Dong, and M. Rege, "Gene expression clustering: A novel graph partitioning approach," in Proceedings of the 2007 International Joint Conference on Neural Networks, IJCNN 2007, pp. 1542-1547, August 2007.

[101] M. Perez, D. M. Rubin, L. E. Scott, T. Marwala, and W. Stevens, "A Hybrid Fuzzy-SVM classifier, applied to gene expression profiling for automated leukaemia diagnosis," in Proceedings of the 2008 IEEE 25th Convention of Electrical and Electronics Engineers in Israel, IEEEI 2008, pp. 41-45, December 2008.

[102] R. Avogadri, M. Brioschi, F. Ferrazzi, M. Re, A. Beghini, and G. Valentini, "A stability-based algorithm to validate hierarchical clusters of genes," International Journal of Knowledge Engineering and Soft Data Paradigms, vol. 1, no. 4, p. 318, 2009.

[103] A. Chaiboonchoe, S. Samarasinghe, and D. Kulasiri, "Using emergent clustering methods to analyse short time series gene expression data from childhood leukemia treated with glucocorticoids," in Proceedings of the 18th World IMACS Congress and International Congress on Modelling and Simulation: Interfacing Modelling and Simulation with Mathematical and Computational Sciences, MODSIM09, pp. 741-747, July 2009.

[104] D. Glez-Peña, F. Díaz, F. Fdez-Riverola, J. R. Méndez, and J. M. Corchado, "Fuzzy patterns and GCS networks to clustering gene expression data," Studies in Fuzziness and Soft Computing, vol. 242, pp. 103-125, 2009.

[105] Y. He and S. C. Hui, "Exploring ant-based algorithms for gene expression data analysis," Artificial Intelligence in Medicine, vol. 47, no. 2, pp. 105-119, 2009.

[106] A. Mukhopadhyay, U. Maulik, and S. Bandyopadhyay, "Unsupervised cancer classification through SVM-boosted multiobjective fuzzy clustering with majority voting ensemble," in Proceedings of the 2009 IEEE Congress on Evolutionary Computation, CEC 2009, pp. 255-261, May 2009.
[107] A. Torkaman, N. M. Charkari, M. Aghaeipour, and E. Hajati, "A recommender system for detection of leukemia based on cooperative game," in Proceedings of the 2009 17th Mediterranean Conference on Control and Automation (MED), pp. 1126-1130, Thessaloniki, Greece, June 2009.

[108] Z. Zheng, S. Sharma, N. Agarwal, and L. Huan, "Integrating Knowledge in Search of Biologically Relevant Genes," in Proceedings of the Data Mining Workshops, 2009 ICDMW'09 IEEE International Conference, W. Jiangxin and C. Yung, Eds., 2009.

[109] A. M. Mehdi, M. S. Sehgal, A. Zayegh, R. Begg, and A. Manan, "K-means clustering on 3rd order polynomial based normalization of Acute Myeloid Leukemia (AML) and Acute Lymphocyte Leukemia (ALL)," in Proceedings of the 2009 3rd International Conference on Electrical Engineering, ICEE 2009, April 2009.

[110] C. Porzelius, M. Johannes, H. Binder, and T. Beissbarth, "Leveraging external knowledge on molecular interactions in classification methods for risk prediction of patients," Biometrical Journal, vol. 53, no. 2, pp. 190-201, 2011.

[111] J. Chen, D. Li, B. Zhang, Z. Wang, Y. Wang, and Q. Zheng, "Notice of Retraction Alternol Inhibits Proliferation and Induces Apoptosis in Human Leukemia HL-60 Cells," in Proceedings of the 2011 5th International Conference on Bioinformatics and Biomedical Engineering, pp. 10-12, 2011.

[112] J. A. Gonzalez, I. Olmos, L. Altamirano et al., "Leukemia identification from bone marrow cells images using a machine vision and data mining strategy," Intelligent Data Analysis, vol. 15, no. 3, pp. 443-462, 2011.

[113] D. L. Tong and A. C. Schierz, "Hybrid genetic algorithm-neural network: Feature extraction for unpreprocessed microarray data," Artificial Intelligence in Medicine, vol. 53, no. 1, pp. 47-56, 2011.

[114] P. S. Chauhan, R. Ihsan, A. K. Mishra et al., "High order interactions of xenobiotic metabolizing genes and P53 codon 72 polymorphisms in acute leukemia," Environmental and Molecular Mutagenesis, vol. 53, no. 8, pp. 619-630, 2012.

[115] H. J. Escalante, M. Montes-y-Gómez, J. A. González et al., "Acute leukemia classification by ensemble particle swarm model selection," Artificial Intelligence in Medicine, vol. 55, no. 3, pp. 163-175, 2012.

[116] K. Y. Yeung, T. A. Gooley, A. Zhang, A. E. Raftery, J. P. Radich, and V. G. Oehler, "Predicting relapse prior to transplantation in chronic myeloid leukemia by integrating expert knowledge and expression data," Bioinformatics, vol. 28, no. 6, Article ID bts059, pp. 823-830, 2012.

[117] T. Manninen, H. Huttunen, P. Ruusuvuori, and M. Nykter, "Leukemia Prediction Using Sparse Logistic Regression," PLoS ONE, vol. 8, no. 8, Article ID e72932, 2013.

[118] A. A. El-Nasser, M. Shaheen, and H. El-Deeb, "Enhanced leukemia cancer classifier algorithm," in Proceedings of the 2014 Science and Information Conference, SAI 2014, pp. 422-429, August 2014.

[119] V. Singhal and P. Singh, "Correlation based feature selection for diagnosis of acute lymphoblastic leukemia," in Proceedings of the 3rd ACM International Symposium on Women in Computing and Informatics (WCI '15), pp. 5-9, Kochi, India, August 2015.

[120] D. Yao, J. Yang, X. Zhan, X. Zhan, and Z. Xie, "A novel random forests-based feature selection method for microarray expression data analysis," International Journal of Data Mining and Bioinformatics, vol. 13, no. 1, pp. 84-101, 2015. 
[121] J. Rawat, A. Singh, H. S. Bhadauria, and J. Virmani, "Computer Aided Diagnostic System for Detection of Leukemia Using Microscopic Images," in Proceedings of the 4th International Conference on Eco-friendly Computing and Communication Systems, ICECCS 2015, pp. 748-756, December 2015.

[122] S. Kar, K. D. Sharma, and M. Maitra, "Gene selection from microarray gene expression data for classification of cancer subgroups employing PSO and adaptive K-nearest neighborhood technique," Expert Systems with Applications, vol. 42, no. 1, pp. 612-627, 2015.

[123] J. Li, Y. Wang, Y. Cao, and C. Xu, "Weighted doubly regularized support vector machine and its application to microarray classification with noise," Neurocomputing, vol. 173, pp. 595605, 2016.

[124] A. K. Dwivedi, "Artificial neural network model for effective cancer classification using microarray gene expression data," Neural Computing and Applications, pp. 1-10, 2016.

[125] S. Krappe, T. Wittenberg, T. Haferlach, and C. Münzenmayer, "Automated morphological analysis of bone marrow cells in microscopic images for diagnosis of leukemia: Nucleus-plasma separation and cell classification using a hierarchical tree model of hematopoesis," in Proceedings of the Medical Imaging 2016: Computer-Aided Diagnosis, March 2016.

[126] R. Ocampo-Vega, G. Sanchez-Ante, M. A. De Luna, R. Vega, L. E. Falcón-Morales, and H. Sossa, "Improving pattern classification of DNA microarray data by using PCA and Logistic Regression," Intelligent Data Analysis, vol. 20, no. 1, pp. S53-S67, 2016.

[127] B. Rajwa, P. K. Wallace, E. A. Griffiths, and M. Dundar, "Automated Assessment of Disease Progression in Acute Myeloid Leukemia by Probabilistic Analysis of Flow Cytometry Data," IEEE Transactions on Biomedical Engineering, vol. 64, no. 5, pp. 1089-1098, 2017.

[128] W. Ni, B. Hu, C. Zheng et al., "Automated analysis of acute myeloid leukemia minimal residual disease using a support vector machine," Oncotarget, vol. 7, no. 44, pp. 71915-71921, 2016.

[129] D. Milojkovic, E. Nicholson, J. F. Apperley et al., "Early prediction of success or failure of treatment with second-generation tyrosine kinase inhibitors in patients with chronic myeloid leukemia," Haematologica, vol. 95, no. 2, pp. 224-231, 2010.

[130] D. White, V. Saunders, A. B. Lyons et al., "In vitro sensitivity to imatinib-induced inhibition of ABL kinase activity is predictive of molecular response in patients with de novo CML," Blood, vol. 106, no. 7, pp. 2520-2526, 2005.

[131] D. L. White and T. P. Hughes, "Predicting the response of CML patients to tyrosine kinase inhibitor therapy," Current Hematologic Malignancy Reports, vol. 4, no. 2, pp. 59-65, 2009.

[132] D. L. White, V. A. Saunders, P. Dang et al., "Most CML patients who have a suboptimal response to imatinib have low OCT-1 activity: Higher doses of imatinib may overcome the negative impact of low OCT-1 activity," Blood, vol. 110, no. 12, pp. 40644072, 2007.

[133] D. L. White, P. Dang, J. Engler et al., "Functional activity of the OCT-1 protein is predictive of long-term outcome in patients with chronic-phase chronic myeloid leukemia treated with imatinib," Journal of Clinical Oncology, vol. 28, no. 16, pp. 2761-2767, 2010.

[134] S. Branford, D. T. Yeung, D. M. Ross et al., "Early molecular response and female sex strongly predict stable undetectable BCR-ABL1, the criteria for imatinib discontinuation in patients with CML," Blood, vol. 121, no. 19, pp. 3818-3824, 2013.
[135] K. Sweet, L. Zhang, and J. Pinilla-Ibarz, "Biomarkers for determining the prognosis in chronic myelogenous leukemia," Journal of Hematology and Oncology, vol. 6, no. 1, article 54, 2013.

[136] W. Prejzner, "Relationship of the BCR gene breakpoint and the type of $\mathrm{BCR} / \mathrm{ABL}$ transcript to clinical course, prognostic indexes and survival in patients with chronic myeloid leukemia," Medical Science Monitor, vol. 8, no. 5, pp. BR193BR197, 2002.

[137] D. Verma, H. M. Kantarjian, D. Jones et al., "Chronic myeloid leukemia (CML) with $\mathrm{P} 190^{B C R-A B L}$ : analysis of characteristics, outcomes, and prognostic significance," Blood, vol. 114, no. 11, pp. 2232-2235, 2009.

[138] J.-Z. Xu and C.-W. Wong, "Hunting for robust gene signature from cancer profiling data: Sources of variability, different interpretations, and recent methodological developments," Cancer Letters, vol. 296, no. 1, pp. 9-16, 2010.

[139] Y.-J. Fan and W. A. Chaovalitwongse, "Optimizing feature selection to improve medical diagnosis," Annals of Operations Research, vol. 174, no. 1, pp. 169-183, 2010.

[140] Z. Chen, J. Li, L. Wei, W. Xu, and Y. Shi, "Multiple-kernel SVM based multiple-task oriented data mining system for gene expression data analysis," Expert Systems with Applications, vol. 38, no. 10, pp. 12151-12159, 2011.

[141] E. J. Yeoh, M. E. Ross, S. A. Shurtleff et al., "Classification, subtype discovery, and prediction of outcome in pediatric acute lymphoblastic leukemia by gene expression profiling," Cancer Cell, vol. 1, no. 2, pp. 133-143, 2002.

[142] A. Mukhopadhyay, U. Maulik, and S. Bandyopadhyay, "Refining genetic algorithm based fuzzy clustering through supervised learning for unsupervised cancer classification," Lecture Notes in Computer Science (including subseries Lecture Notes in Artificial Intelligence and Lecture Notes in Bioinformatics), vol. 5483, pp. 191-202, 2009.

[143] J.-H. Cho, D. Lee, H. P. Jin, K. Kim, and I.-B. Lee, "Optimal approach for classification of acute leukemia subtypes based on gene expression data," Biotechnology Progress, vol. 18, no. 4, pp. 847-854, 2002.

[144] Z. Chen, J. Li, and L. Wei, "A multiple kernel support vector machine scheme for feature selection and rule extraction from gene expression data of cancer tissue," Artificial Intelligence in Medicine, vol. 41, no. 2, pp. 161-175, 2007.

[145] M. Baccarani, G. Saglio, J. Goldman et al., "Evolving concepts in the management of chronic myeloid leukemia: recommendations from an expert panel on behalf of the European LeukemiaNet," Blood, vol. 108, no. 6, pp. 1809-1820, 2006.

[146] T. Hughes and S. Branford, "Molecular monitoring of BCR$\mathrm{ABL}$ as a guide to clinical management in chronic myeloid leukaemia," Blood Reviews, vol. 20, no. 1, pp. 29-41, 2006.

[147] M. Baccarani, G. Gugliotta, F. Castagnetti, S. Soverini, and G. Rosti, "A Review and an Update of European LeukemiaNet Recommendations for the Management of Chronic Myeloid Leukemia," in Chronic Myeloid Leukemia, Hematologic Malignancies, pp. 55-69, Springer International Publishing, Cham, 2016.

[148] S. L. Noble, E. Sherer, R. E. Hannemann, D. Ramkrishna, T. Vik, and A. E. Rundell, "Using adaptive model predictive control to customize maintenance therapy chemotherapeutic dosing for childhood acute lymphoblastic leukemia," Journal of Theoretical Biology, vol. 264, no. 3, pp. 990-1002, 2010. 
[149] L. M. Fagan and E. H. Shortliffe, The Future of Computer Applications in Biomedicine. Biomedical Informatics Computer Applications in Health Care and Biomedicine, Health Informatics, Springer, New York, NY, USA, 3rd edition, 2006.

[150] J. A. Cruz and D. S. Wishart, "Applications of machine learning in cancer prediction and prognosis," Cancer Informatics, vol. 2, pp. $59-77,2006$. 


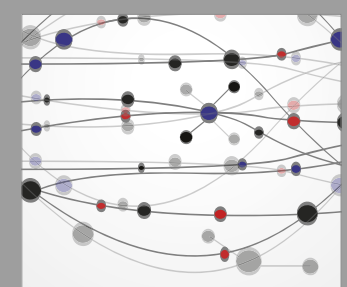

The Scientific World Journal
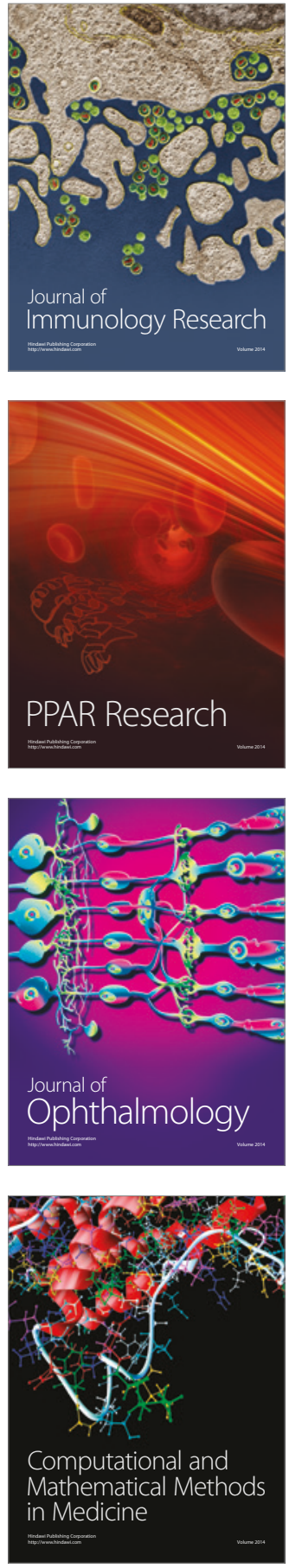

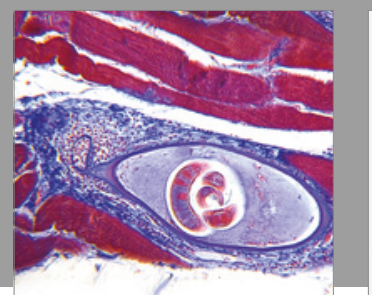

Gastroenterology Research and Practice
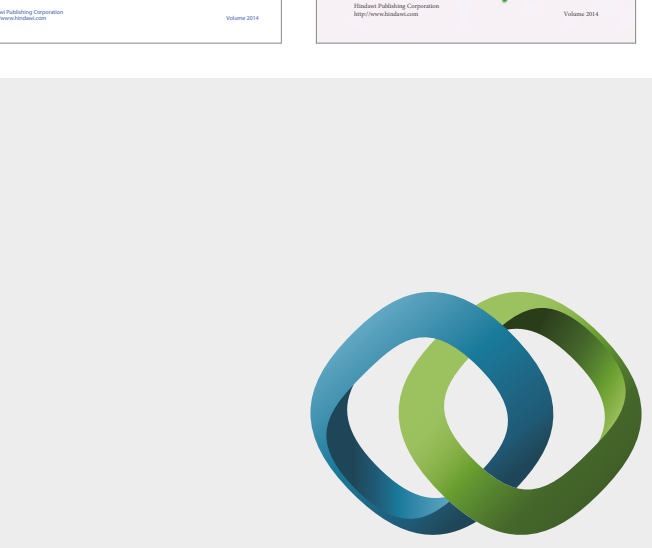

\section{Hindawi}

Submit your manuscripts at

https://www.hindawi.com
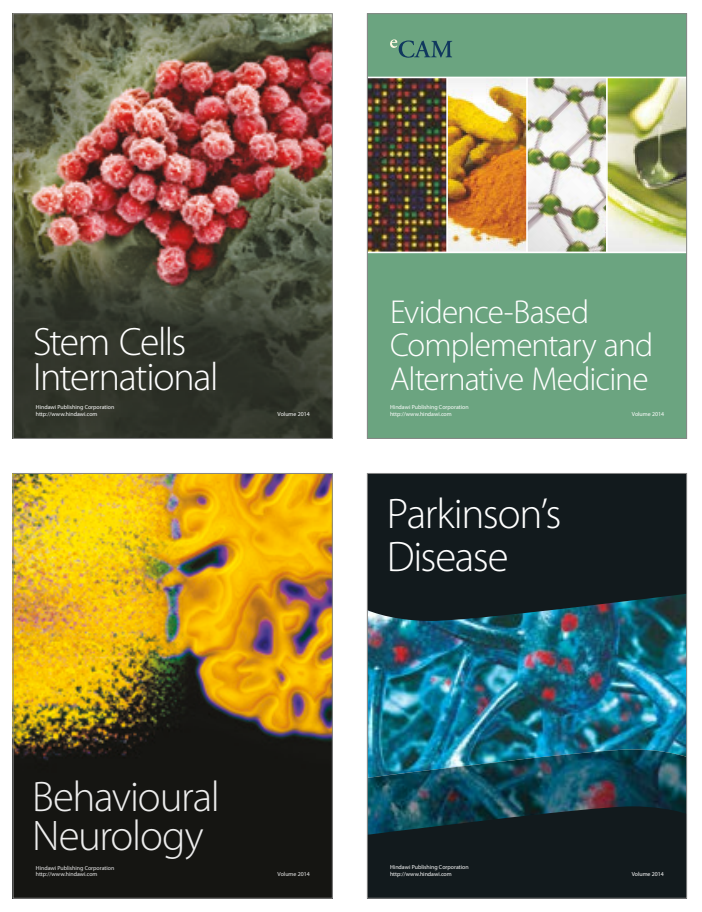
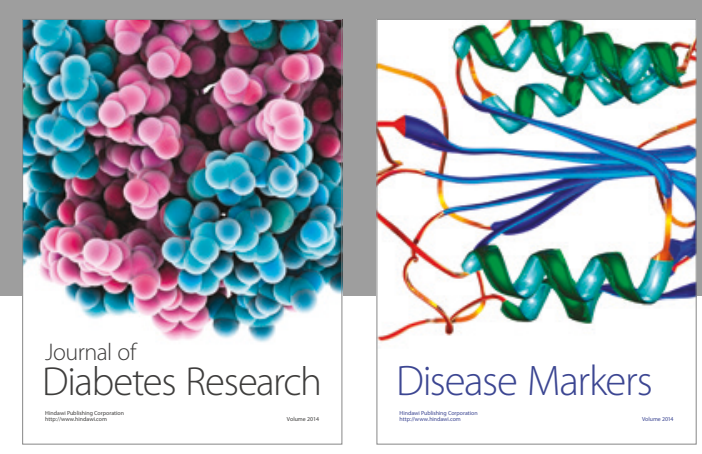

Disease Markers
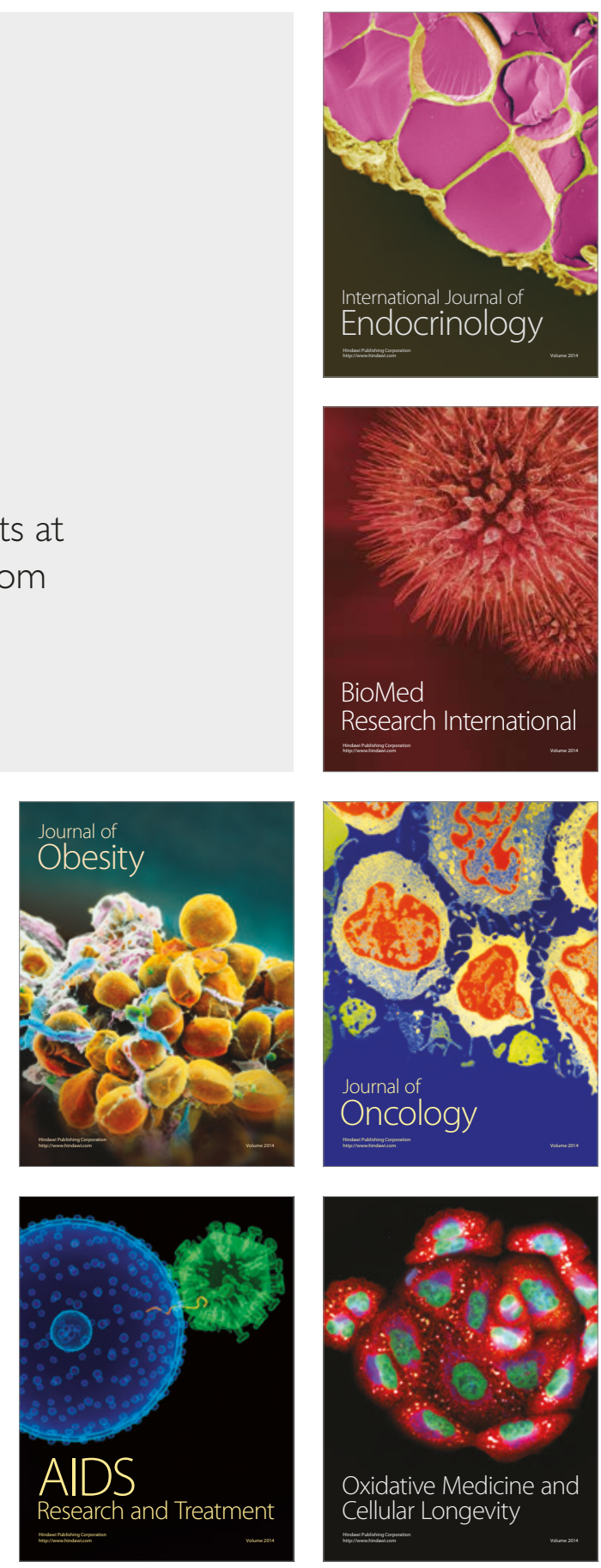